\title{
Central role for GSK3 $\beta$ in the pathogenesis of arrhythmogenic cardiomyopathy
}

\author{
Stephen P. Chelko, ${ }^{1}$ Angeliki Asimaki, ${ }^{2}$ Peter Andersen, ${ }^{1}$ Djahida Bedja, ${ }^{1,3}$ Nuria Amat-Alarcon, ${ }^{1}$ \\ Deeptankar DeMazumder, ${ }^{1}$ Ravirasmi Jasti, ${ }^{1}$ Calum A. MacRae, ${ }^{2,4}$ Remo Leber, ${ }^{5}$ Andre G. Kleber, ${ }^{2}$ \\ Jeffrey E. Saffitz, ${ }^{2}$ and Daniel P. Judge ${ }^{1}$ \\ 'Department of Medicine/Cardiology, Johns Hopkins University School of Medicine, Baltimore, Maryland, USA. \\ 2Department of Pathology, Beth Israel Deaconess Medical Center and Harvard Medical School, Boston, \\ Massachusetts, USA. ${ }^{3}$ Australian School of Advanced Medicine, Macquarie University, Sydney, New South Wales, \\ Australia. ${ }^{4}$ Department of Medicine, Brigham and Women's Hospital and Harvard Medical School, Boston, \\ Massachusetts, USA. ${ }^{5}$ Schiller AG, Research and Development, Baar, Switzerland.
}

\begin{abstract}
Arrhythmogenic cardiomyopathy (ACM) is characterized by redistribution of junctional proteins, arrhythmias, and progressive myocardial injury. We previously reported that SB216763 (SB2), annotated as a CSK3 $\beta$ inhibitor, reverses disease phenotypes in a zebrafish model of ACM. Here, we show that SB2 prevents myocyte injury and cardiac dysfunction in vivo in two murine models of ACM at baseline and in response to exercise. SB2-treated mice with desmosome mutations showed improvements in ventricular ectopy and myocardial fibrosis/inflammation as compared with vehicle-treated (Veh-treated) mice. CSK3 $\beta$ inhibition improved left ventricle function and survival in sedentary and exercised $D s g 2^{\text {mut } / \text { mut }}$ mice compared with Veh-treated Dsg2 ${ }^{\text {mut } / \text { mut }}$ mice and normalized intercalated disc (ID) protein distribution in both mutant mice. CSK3 $\beta$ showed diffuse cytoplasmic localization in control myocytes but ID redistribution in ACM mice. Identical CSK3 $\beta$ redistribution is present in ACM patient myocardium but not in normal hearts or other cardiomyopathies. SB2 reduced total CSK3 $\beta$ protein levels but not phosphorylated Ser 9-CSK3 $\beta$ in ACM mice. Constitutively active CSK3 $\beta$ worsens ACM in mutant mice, while CSK3 $\beta$ shRNA silencing in ACM cardiomyocytes prevents abnormal ID protein distribution. These results highlight a central role for GSK $\beta$ in the complex phenotype of ACM and provide further evidence that pharmacologic CSK $\beta$ inhibition improves cardiomyopathies due to desmosome mutations.
\end{abstract}

Authorship note: S.P. Chelko and A. Asimaki contributed equally to this work.

Conflict of interest: The authors have declared that no conflict of interest exists.

Submitted: December 14, 2015 Accepted: March 18, 2016 Published: April 21, 2016

Reference information: JCI Insight. 2016;1(5):e85923. doi:10.1172/jici.nsight.85923.

\section{Introduction}

Arrhythmogenic cardiomyopathy (ACM) is an inherited form of heart disease, characterized clinically by ventricular dysfunction and arrhythmias and pathologically by fibrofatty scar (1). Mutations in genes encoding elements of the cardiac desmosome are the most frequent cause, although pathogenic mechanisms are poorly understood. Life-threatening ventricular arrhythmias occur frequently, and heart failure also develops in later stages, with progressive cardiac dysfunction. The right ventricle is often disproportionately affected. Prior investigation identified a reduction in canonical Wnt signaling in ACM, implicating this pathway in its pathogenesis (2).

We previously created a zebrafish model of ACM, which we used in a chemical screen to identify a small molecule (SB216763 [SB2]) that rescues the disease phenotype (3). SB2 also reverses features of ACM in neonatal rat ventricular myocytes (NRVMs) expressing disease alleles and in cardiomyocytes produced from induced pluripotent stem cells from patients with ACM (3). SB2 is annotated as an inhibitor of $\operatorname{GSK} 3 \beta(4,5)$, thereby increasing canonical Wnt signaling. Its efficacy in vivo in mammalian models of ACM has not previously been demonstrated.

Recent studies have revealed the dichotomy of acute and long-term inhibition of GSK3 $\beta$ on cardiac function and remodeling via genetically modified animal models or pharmacological inhibition (6). Under normal conditions, inhibition of GSK3 $\beta$ potentiates Wnt signaling and disrupts cardiac homeostasis, with consequently increased myocardial fibrosis, left ventricle (LV) dysfunction, and hypertrophy (7). In contrast, inhibition of GSK3 $\beta$ during pathological conditions, such as animal models of myocardial infarction (8), ischemia/reperfusion (9), and pressure overload (10), results in cardioprotection via reduced myocardial apoptosis, fibrosis, and LV dysfunction. Here, we demonstrate that GSK3 $\beta$ is uniquely mislocalized in 
both mice and people with ACM; its inhibition with SB2 improves cardiac histology, function, and arrhythmia at rest and in response to exercise in murine models of ACM; and that modulation of this kinase coordinately alters the course of ACM. These results strongly suggest that a common disease mechanism involving aberrant GSK3 $\beta$ signaling is responsible for the complex clinical phenotype in ACM. They also suggest that the salutary effects of SB2 are mediated by inhibition of GSK3 $\beta$.

\section{Results}

Targeting of murine Dsg2, encoding desmoglein-2, recapitulates ACM. We previously reported that mutations in human DSG2 contribute to the development of ACM (11). Homologous recombination in murine embryonic stem cells was used to insert a targeting vector with FRT and loxP sites flanking exons 4 and 5 in murine Dsg2 (Figure 1A). Homozygous targeted mice without Flp and Cre recombinase (Dsg2 ${ }^{\text {neo/neo }}$ ) had a normal cDNA sequence but with reduced transcript abundance ( $15 \%$ compared with the untargeted allele). $D s g 2^{\text {neo/neo }}$ mice developed cardiomyopathy by 16 weeks of age (Supplemental Figure 1, A-C; supplemental material available online with this article; doi:10.1172/jci.insight.85923DS1), supporting loss-of-function rather than gain-of-function or dominant-negative interference as the predominant mechanism. The neomycin selection cassette was removed by Flp-mediated recombinase expression via mating, followed by excision of exons 4 and 5 via Cre-mediated recombinase mating ( $D s g 2^{\text {mut } / \text { mut }}$, Figure 1A). Loss of exons 4 and 5 caused a frameshift mutation and premature truncation, which is predicted to be degraded by nonsense-mediated mRNA decay. Heterozygous mice prior to or after Flp/Cre recombination are designated as $D s g 2^{\text {neo/+ }}$ and $D s g 2^{\text {mut/+ }}$, respectively.

Dsg2 $2^{\mathrm{mut} / \mathrm{mut}}$ mice developed cardiomyopathy by 8 weeks of age compared with WT littermates (Figure 1, B and C, and Supplemental Table 1). By 16 weeks of age, ventricular myocardia from $D s g 2^{\text {mut/mut }}$ mice showed an absence of DSG2 protein by Western analysis and complete loss of junctional DSG2 immunostaining (Figure 2A, see complete unedited blots in the supplemental material); abnormal plakoglobin (PLK) and connexin43 (Cx43) signal at myocyte junctions, with normal distribution of N-cadherin (Figure 2B); and extensive biventricular fibrosis compared with WT controls (Figure 2, C-F).

Transgenic myocyte-specific expression of human 2157del2 PLK recapitulates ACM. Mice heterozygous for a null allele in PLK show some features of right ventricular dysfunction, which were only inducible by forced exercise (12). Using the $\alpha$-MHC promoter, we developed a transgenic line with cardiomyocyte-specific expression of PLK with the 2157del2 PLK mutation (JUP ${ }^{2157 d e l 2}$ ) found in Naxos disease (Naxos PLK). Western analysis demonstrated equivalent amounts of mutant and WT PLK (Figure 3A). These animals died at approximately 1 year of age. Hearts from $J U P^{2157 \text { del2 }}$ mice displayed focal areas of inflammation and increased apoptotic cardiomyocytes compared with WT controls (Figure 3B). Immunoreactive signal for PLK was absent at intercalated discs (IDs), and signal for Cx43 and synapse-associated protein 97 (SAP97) was substantially depressed, with control-like N-cadherin distribution (Figure 3C). Despite overproduction of transgenic PLK levels and equivalent endogenous PLK levels in JUP $P^{2157 \mathrm{del} 2}$ mice, immunoreactive signal for PLK was reduced at IDs in $J U P^{2157 d e l 2}$ mice. Serial dilution immunoreactive-studies confirmed predominant cytoplasmic staining in $J U P^{2157 \mathrm{del} 2}$ mice (Supplemental Figure 2, A and B). In previous studies, we observed greatly reduced signals for PLK, Cx43, and SAP97 at IDs in NRVM models of ACM and in induced pluripotent stem cell-derived cardiomyocytes from patients with ACM (3). Similar reductions are seen in ACM patient myocardium $(3,13)$. Thus, this transgenic model (JUP ${ }^{2157 d e l 2}$ ) recapitulates ACM, likely by dominant-negative interference.

SB2 treatment improves features of ACM in Dsg2 $2^{\text {mut } / m u t}$ and JUP 2157 del2 mice. We sought to determine the efficacy of SB2 treatment in two murine models of ACM. Mice were treated daily with SB2 (i.p., $2.5 \mathrm{mg} /$ $\mathrm{kg} / \mathrm{d})$ at 6 months $\left(J U P^{2157 \mathrm{del} 2}\right)$ and 3 weeks $\left(D s g 2^{\mathrm{mut} / \mathrm{mut}}\right)$ of age for $6\left(J U P^{2157 \mathrm{del} 2}\right)$ or 13 (Dsg2 $\left.2^{\mathrm{mut} / \mathrm{mut}}\right)$ weeks. Control mice were administered equivalent volume $/ \mathrm{kg} / \mathrm{d}$ of vehicle (DMSO). Hearts of vehicle-treated $J U P^{2157 \mathrm{del} 2}$ mice exhibited focal areas of fibrosis and inflammation, which were virtually absent in SB2treated mice (Figure 4, A and B). Vehicle-treated Dsg $2^{\mathrm{mut} / \mathrm{mut}}$ mice displayed extensive biventricular epicardial and endocardial fibrosis and inflammation, in contrast with SB2-treated Dsg2 ${ }^{\text {mut } / \text { mut }}$ mice (Figure $4, \mathrm{~A}$ and $\mathrm{B})$. Vehicle-treated $J U P^{2157 \mathrm{del} 2}$ mice exhibited high levels of cardiomyocyte apoptosis, which was eliminated by SB2 (Figure 4, A and B).

To determine whether cardiac contractile and electrical phenotypes could also be reversed by SB2 in vivo, mice were assessed via ECG telemetry ( $J U P^{2157 \mathrm{del} 2}$ and $\left.D s g 2^{\mathrm{mut} / \mathrm{mut}}\right)$ and echocardiography (Dsg2 $\left.2^{\mathrm{mut} / \mathrm{mut}}\right)$. Vehicle-treated $J U P^{2157 \mathrm{del} 2}$ mice exhibited frequent ventricular ectopy and short runs of ventricular tachy- 

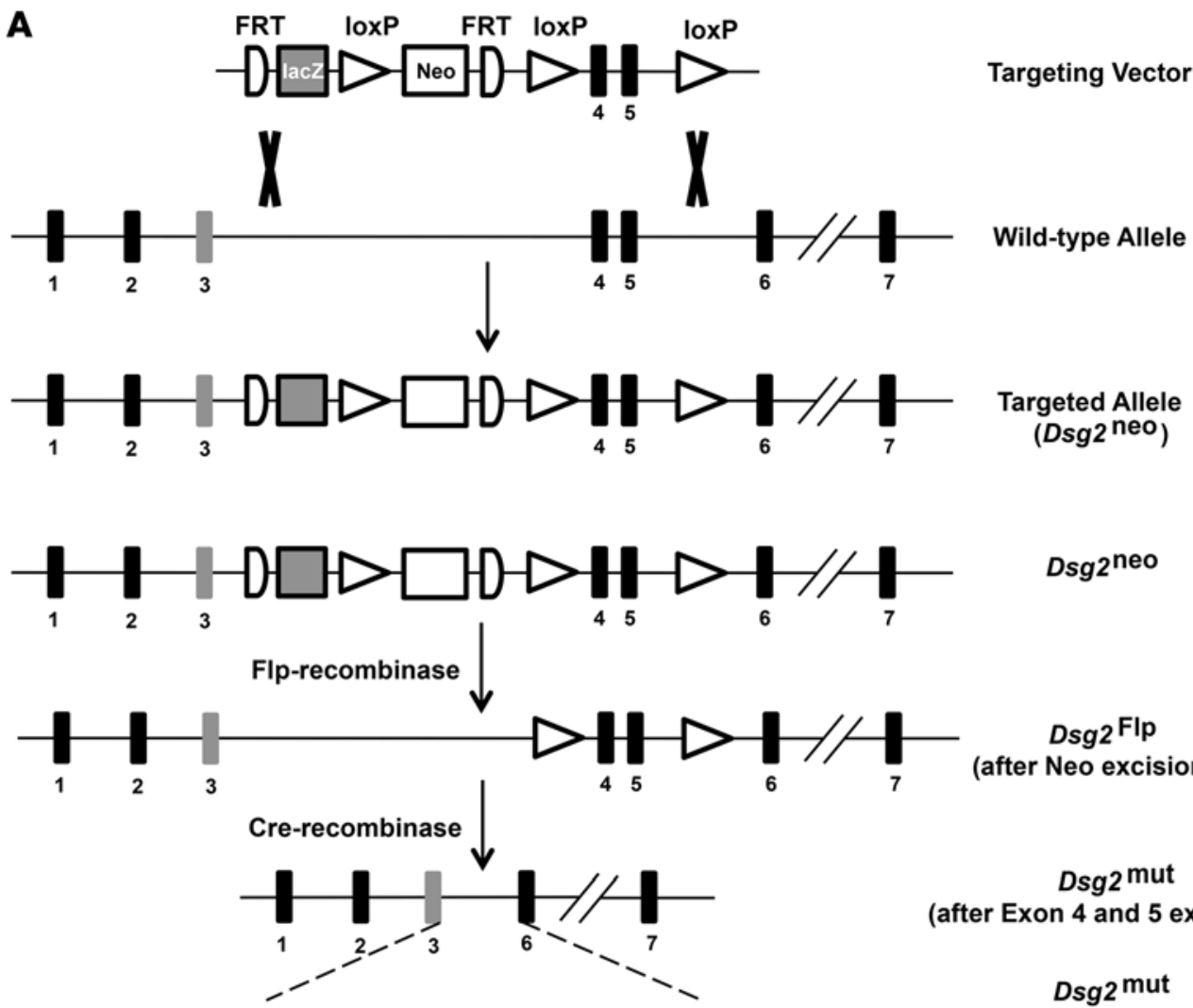

Dsg2neo

ATT GCC AAG ATA CAC TTG TGA* TGA*

(cDNA)

B Short-axis M-mode

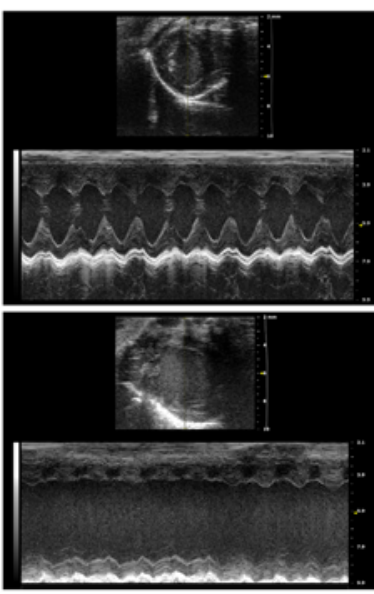

LV Long-axis

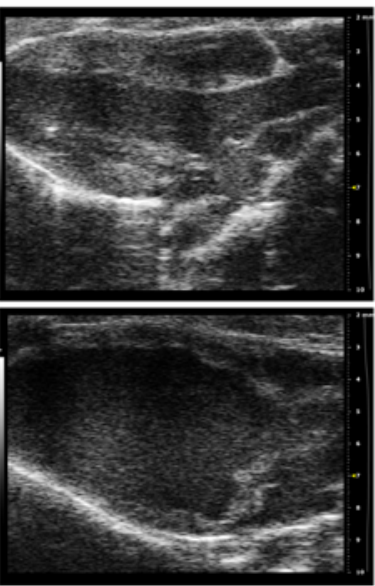

C

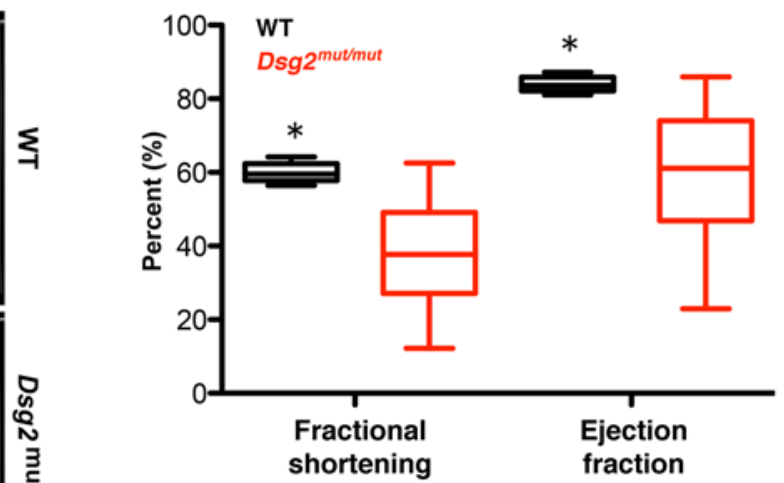

Figure 1. Targeting of murine $D s g 2$, encoding desmoglein-2, recapitulates ACM. (A) Structure of targeting vector for $D s g 2^{\text {mut/mut }}$ mutant mice and $D s g 2^{\text {mut }}$ allele after Flp- and Cre-mediated recombination. Note that Dsg ${ }^{\text {mut }}$ cDNA has two stop codons (TCA ${ }^{\ddagger}$ ) in exon 6. (B) Representative short-axis m-mode and left ventricle (LV) long-axis echocardiography from WT and Dsg $2^{\text {mut/mut }}$ mice at 8 weeks of age. Echocardiographic images are representative of $n=7$ and $n=6$ for WT and Dsg2 $2^{\text {mut/mut }}$ mice, respectively. (C) Decreased fractional shortening and ejection fraction in Dsg2mut/mut mice at 8 weeks of age. Horizontal bars indicate the medians, boxes indicate 25 th to 75 th percentiles, and whiskers indicate 10th and 90th percentiles. Mean \pm SEM, $n \geq 6 /$ genotype, ${ }^{*} P<0.05$ for WT vs. Dsg2 ${ }^{\text {mut/mut }}$ using 2-way ANOVA with Tukey's post-hoc analysis.

cardia (Figure 4C). SB2-treated JUP ${ }^{2157 \mathrm{del} 2}$ mice showed less ventricular ectopy (Figure 4C) compared with vehicle-treated mutant mice. In $D s g 2^{\text {mut } / \text { mut }}$ mice, ejection fraction (EF) and fractional shortening (FS), as measured by echocardiography, were lower by 8 weeks of age compared with those in WT controls (Figure 1 and Supplemental Table 1). In contrast, SB2 treatment resulted in better cardiac function in Dsg $2^{\mathrm{mut} / \mathrm{mut}}$ mice (Figure 4D). Increased ventricular ectopy in $D s g 2^{\mathrm{mut} / \mathrm{mut}}$ mice caused prolongation of the average QRS dura- 
A
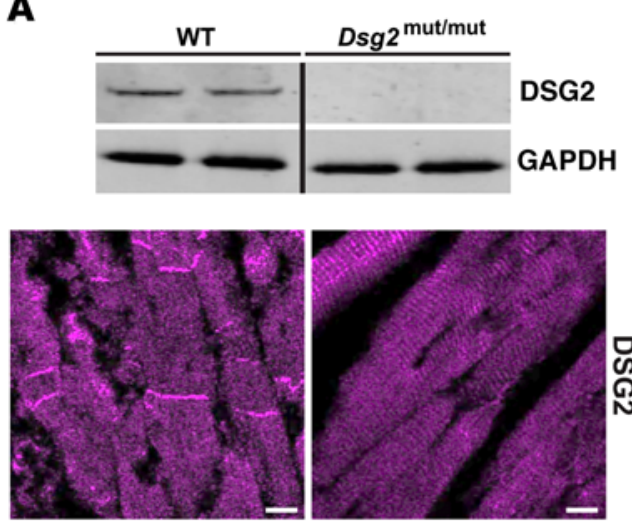

B
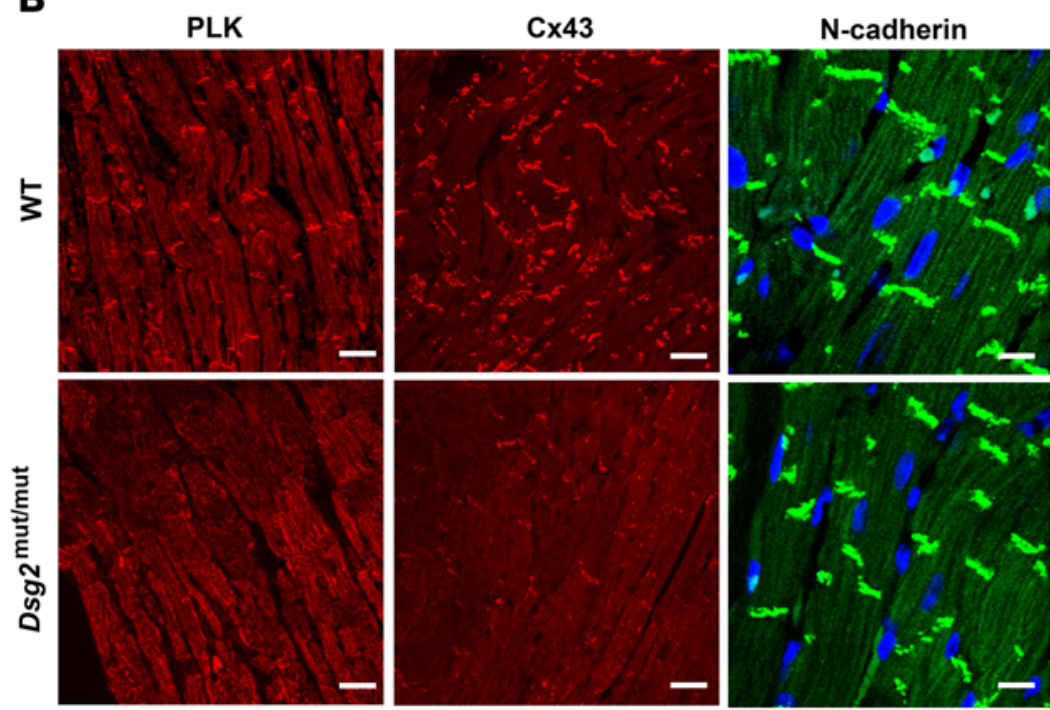

C

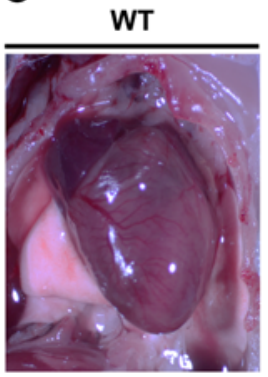

D

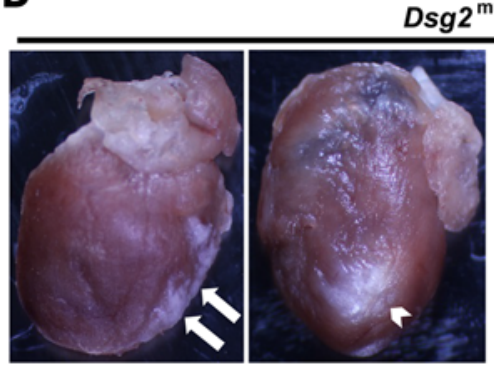

Dsg2 $2^{\text {mut/mut }}$

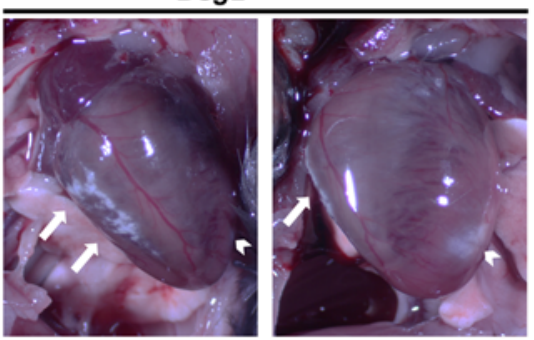

sgg $2^{\text {mut/mut }}$
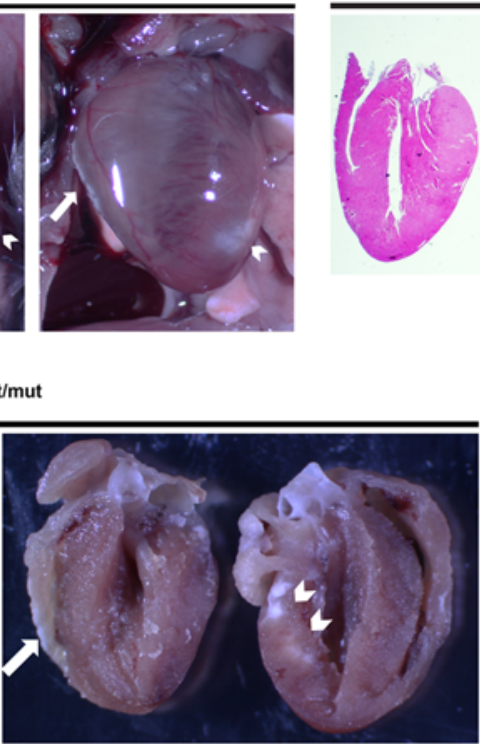

E

MTC

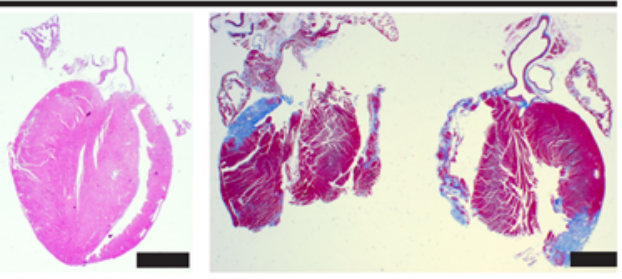

$\mathbf{F}$

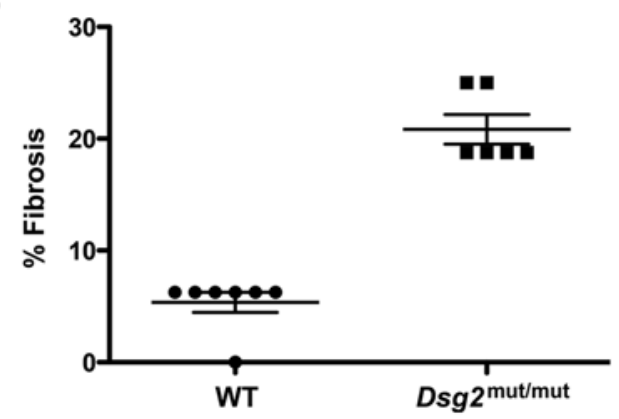

Figure 2. Biventricular fibrosis and abnormal distribution of junctional proteins in Dsg2 ${ }^{\text {mut/mut }}$ mice at 16 weeks of age. (A) Western immunoblots and immunolabeled myocardium probed for desmoglein-2 (DSC2) showed a complete absence of protein and junctional distribution of DSC2 in Dsg2 ${ }^{\text {mut/mut }}$ mice compared with WT mice. Scale bar: $10 \mu \mathrm{m}$. (B) Representative images from myocardium immunolabeled for plakoglobin (PLK), connexin43 (Cx43), and $\mathrm{N}$-cadherin. Scale bar: $20 \mu \mathrm{m}$. (C and D) Gross pathology shows right ventricle (white arrows) and left ventricle (white arrowhead) epicardial and endocardial scars. (E) Masson's trichrome-immunostained (MTC-immunostained) myocardium from Dsg2 ${ }^{\mathrm{mut} / \mathrm{mut}}$ mice shows extensive epicardial and endocardial scarring compared with WT controls. Scale bar: $1 \mathrm{~mm}$. Images are representative of $n=7$ and $n=6$ for WT and Dsg2 $2^{\text {mut/mut }}$ mice, respectively. (F) Percentage fibrosis presented as mean \pm SEM. $P<0.05$ for WT vs. Dsg2 $2^{\text {mut } / \text { mut }}$ using 2-tailed $t$ test with equal variance.

tion (Figure 4D), whereas treatment with SB2 normalized QRS duration in Dsg2 $2^{\text {mut } / \text { mut }}$ mice (Figure 4D).

Abnormal distribution of ID proteins, such as PLK and Cx43, is a hallmark feature of ACM and an early manifestation of disease progression (13). We tested the effects of SB2 treatment on ID protein distribution and total protein levels in $J U P^{2157 \mathrm{del} 2}$ and $D s g 2^{\mathrm{mut} / \mathrm{mut}}$ mice. Total cellular protein lysates and formalin-fixed, paraffin-embedded myocardium were probed for changes in protein levels and cardiomyocyte distribution, respectively (Figure 5, A-D, and Supplemental Figure 3, A and B). Immunolabeled ventricular myocardium from both mutant lines showed a marked loss in junctional signals for PLK, Cx43, and SAP97, all of which were normalized by SB2 (Figure 5, A and B, and Supplemental Figure 3A). Changes 
A

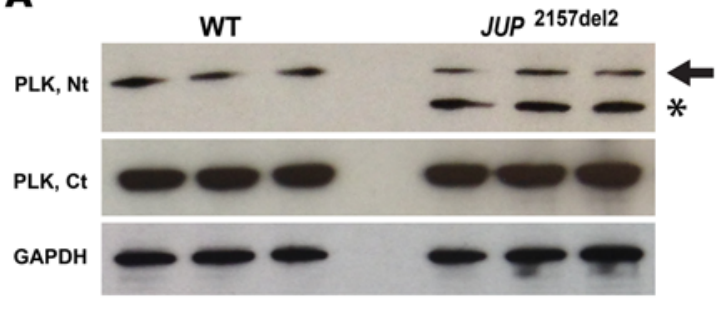

B
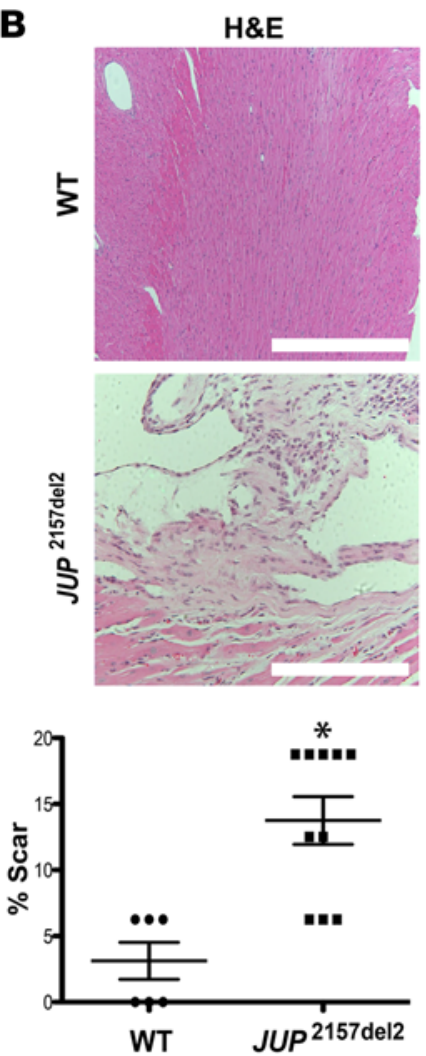

TUNEL
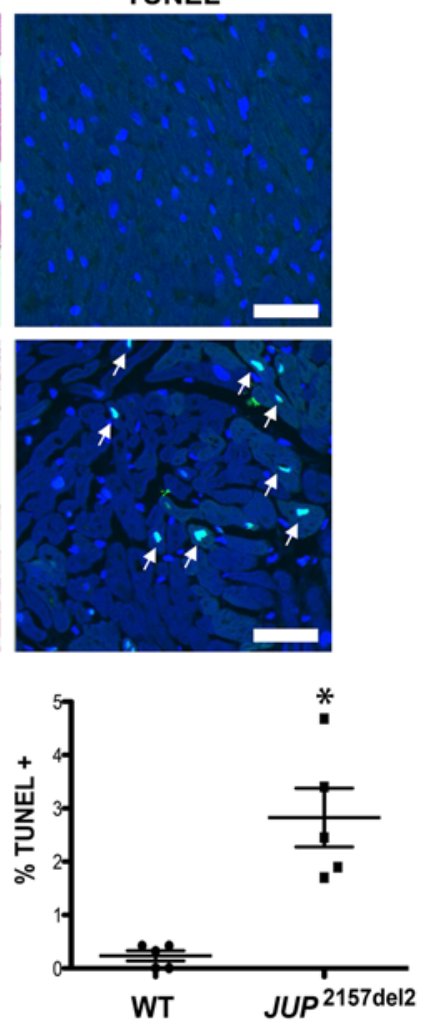

C
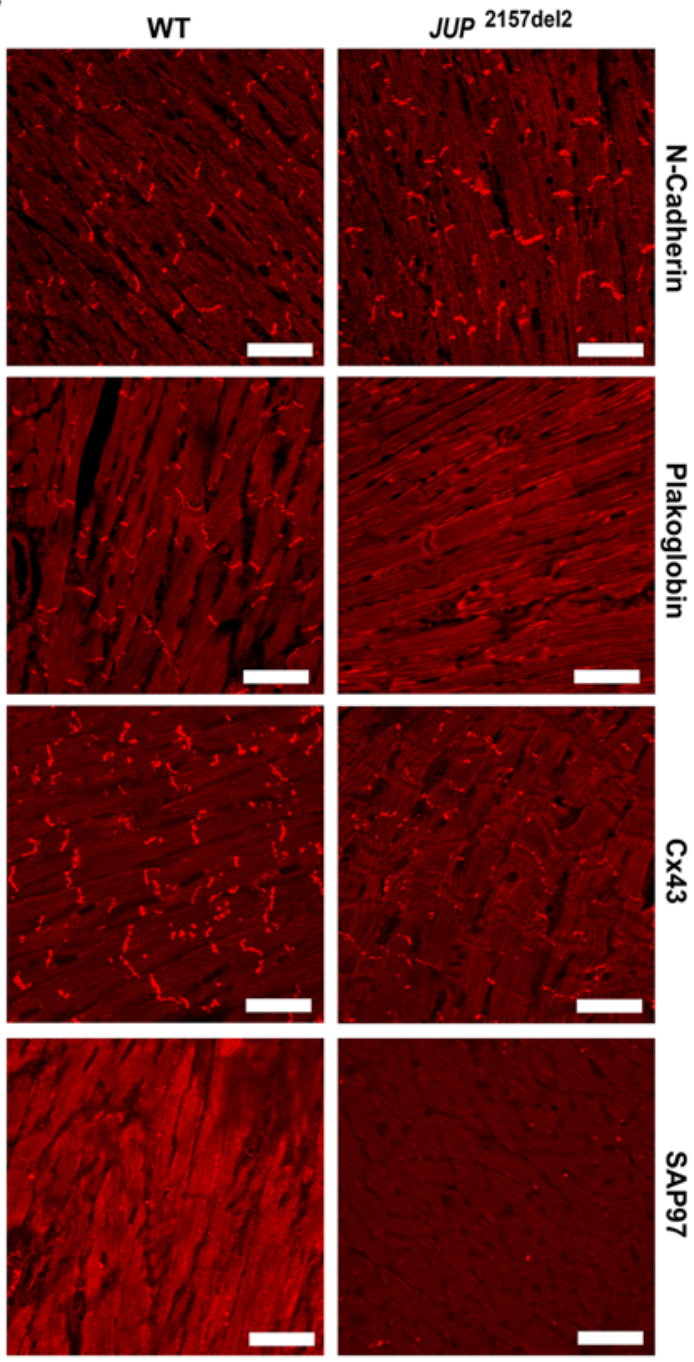

Figure 3. Transgenic myocyte-specific expression of human 2157del2 plakoglobin recapitulates ACM. (A) Representative Western immunoblots from WT and JUP 2157 del2 mouse hearts. Antibodies directed against the plakoglobin N-terminus (PLK, Nt) detect both the endogenous PLK (black arrowhead) and truncated mutant transgene (black asterisk). Antibodies directed against the C-terminus (PLK, Ct) do not detect the mutant transgenic protein and confirm that endogenous Jup expression is not reduced in transgenic mice compared with WT. $n=3 /$ genotype. (B) JUP ${ }^{2157 d e l 2}$ hearts display focal areas of inflammation (scale bar: $100 \mu \mathrm{m}$ ) and increased TUNEL-positive nuclei (white arrowheads; scale bar: $20 \mu \mathrm{m}$ ). H\&E: $n=6$ for WT and $n=10$ for JUP2157del2; TUNEL: $n=5$ /genotype. The percentage of scarring and the percentage of TUNEL-positive nuclei are presented as mean \pm SEM. ${ }^{*} P<0.05$ for WT vs. JUP $P^{2157 d 2}$ using 2-tailed $t$ test with equal variance. (C) Representative images from JUP $P^{2157 d 2}$ and WT control hearts immunolabeled for N-cadherin, plakoglobin, connexin43 (Cx43), and synapse-associated protein 97 (SAP97). Images are representative of $n \geq 5$ /genotype. Scale bar: $20 \mu \mathrm{m}$.

in protein distribution occurred without an apparent change in PLK, desmoplakin, and SAP97 protein levels, as shown by Western immunoblotting (Figure 5 and Supplemental Figure 3, A and B). SB2 treatment was associated with increased $\mathrm{Cx} 43$ protein levels in both mutant lines (Figure 5, C and D), consistent with previous studies that demonstrated that activation of Wnt signaling increases $\mathrm{Cx} 43$ expression (14).

GSK3 $\beta$ localization is uniquely abnormal in ACM, and SB2 normalizes it. The cytosolic Axin-APC-GSK3 $\beta$ complex targets $\beta$-catenin for degradation via phosphorylation of Ser/Thr residues, first by the priming kinase, casein kinase-1 at Ser45, and subsequently by GSK3 $\beta$ at Ser33/37/Thr41 (15), and SB2 is annotated as an inhibitor of $\operatorname{GSK} 3 \beta(4,5)$. Because of the remarkably favorable responses to SB2 in ACM models, we reasoned that GSK3 $\beta$ might play a central role in disease pathogenesis. In WT mice, cardiac immunohistochemistry demonstrated that GSK3 $\beta$ is distributed in a diffuse cytoplasmic pattern (Figure 6A). In contrast, GSK $3 \beta$ signal was predominantly located at the myocyte junction in both murine models of ACM (Figure 6A). NRVMs expressing mutant forms of PLK (JUP $P^{2157 \text { del2 }}$ ) or plakophilin-2 (PKP2 $\left.2^{1851 \text { del123 }}\right)$ demon- 
A
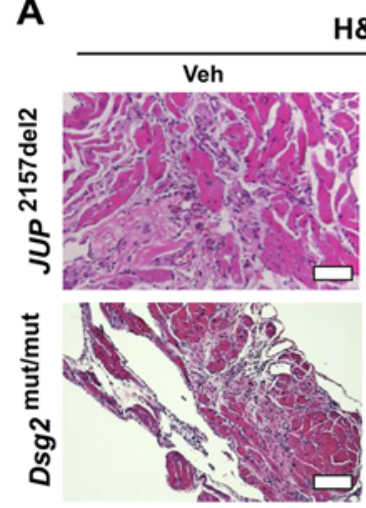

B
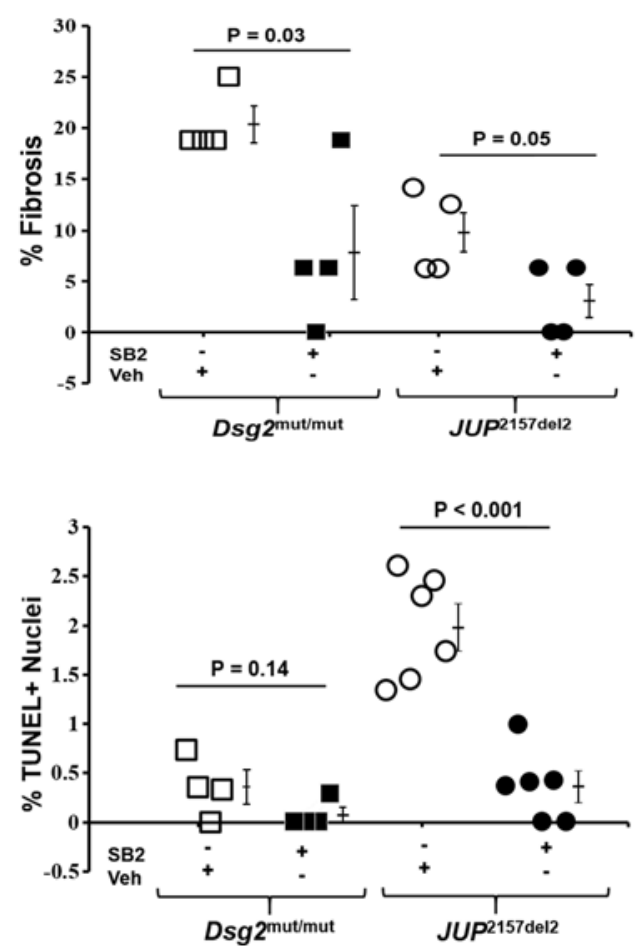

MTC
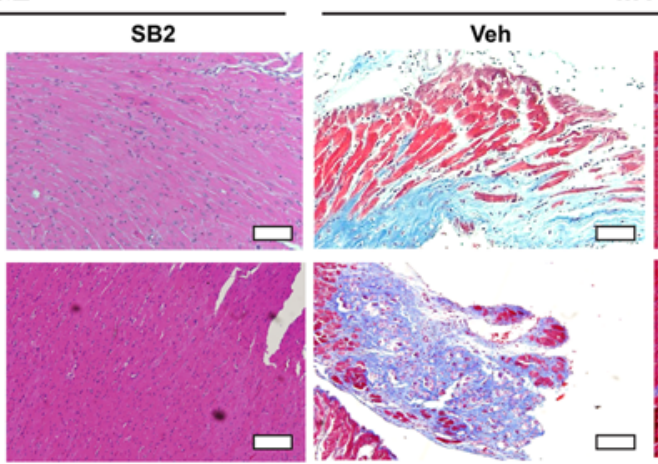

C

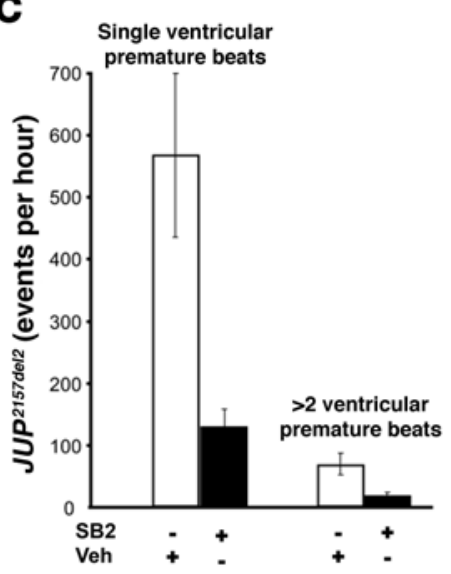

Ventricular premature beat, type 1

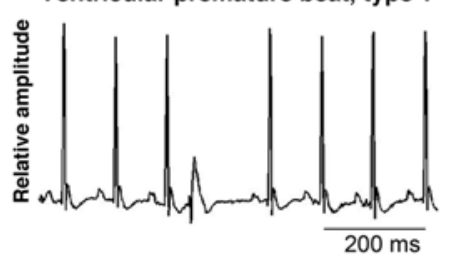

D
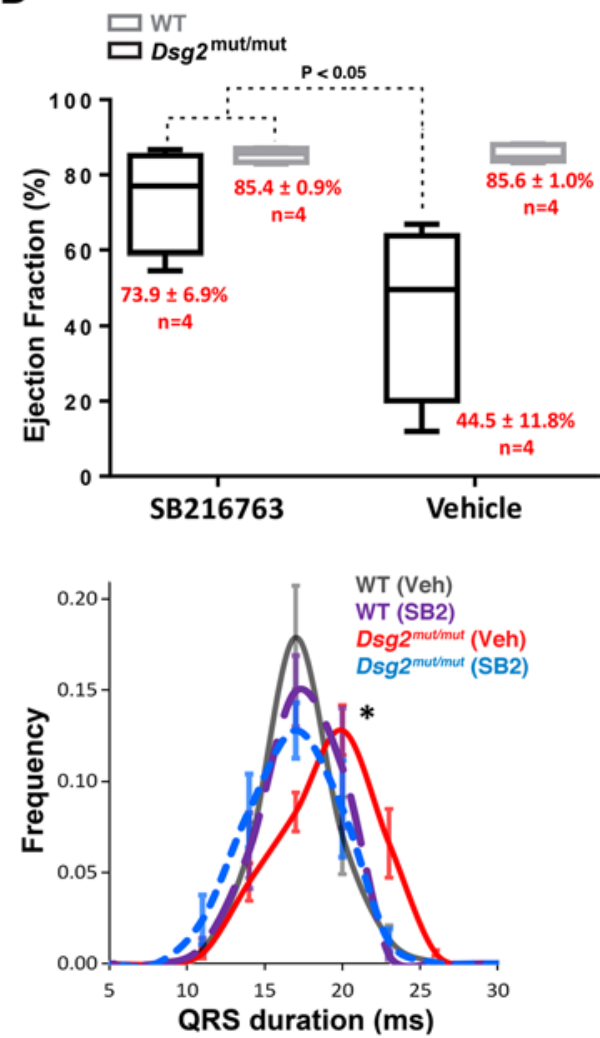

Figure 4. SB216763 treatment improves myocardial injury and cardiac function in JUP2157del2 and Dsg2 ${ }^{\text {mut/mut }}$ mice. (A) Representative images of ventricular myocardia from JUP2157del2 and Dsg2mut/mut mice treated with vehicle (Veh) or SB216763 (SB2). Myocardia were immunostained with H\&E, Masson's trichrome (MTC), and TUNEL. Scale bar: $50 \mu \mathrm{m}$. Images are representative of $n \geq 4$ /genotype (white arrows, TUNEL-positive nuclei). (B) Percentage fibrosis and TUNEL-positive nuclei in hearts from SB2- and Veh-treated mutant mice. Fibrosis: $n=4 /$ genotype; TUNEL: $n=4$ for WT and Dsg2mut/mut mice and $n=6$ for WT and JUP2157del2 mice. Mean \pm SEM. $P<0.05$ for WT vs. ACM mice using 2-tailed paired $t$ test. (C) Quantitative ECG telemetry analysis of SB2-treated JUP $P^{2157 \mathrm{de} 2}$ mice exhibited decreased bouts of single and $>2$ premature ventricular complexes. Representative ECC telemetry tracing from a vehicle-treated JUP 2157del2 mouse. Mean \pm SEM. $P<0.05$ for SB2-treated JUP2157del2 $(n=16)$ vs. Veh-treated JUP2157del2 $(n=6)$ mice using 2-tailed $t$ test with equal variance. (D) Echocardiography and ECG telemetry analysis of Veh- and SB2-treated Dsg2 ${ }^{\mathrm{mut} / m u t}$ mice. In box-and-whisker plots, horizontal bars indicate the medians, boxes indicate 25 th to 75 th percentiles, and whiskers indicate 10 th and 90th percentiles. Mean $\pm \mathrm{SEM}, n=4 /$ genotype/treatment. ${ }^{*} P<0.05$ for SB2treated $D s g 2^{\text {mut/mut }}$ vs. Veh-treated $D s g 2^{\text {mut/mut }}$ mice using 2-way ANOVA with Tukey's post-hoc analysis.

strated similar distribution of GSK3 $\beta$ at IDs, whereas a diffuse cytoplasmic pattern was observed in control untransfected NRVM cells (Figure 6A). In order to determine whether the pattern of abnormal GSK3 $\beta$ immunostaining is affected by the longitudinal shape of myocytes, we utilized a microprinting method to insure a longitudinal pattern of NRVMs. Abnormal GSK3 $\beta$ immunoreactive signal at IDs was observed in patterned longitudinal ACM mutant NRVMs compared with untreated control NRVMs (Supplemental Figure 4). The accumulation of GSK3 $\beta$ immunostaining at the ID in mutant patterned NRVMs was 
A
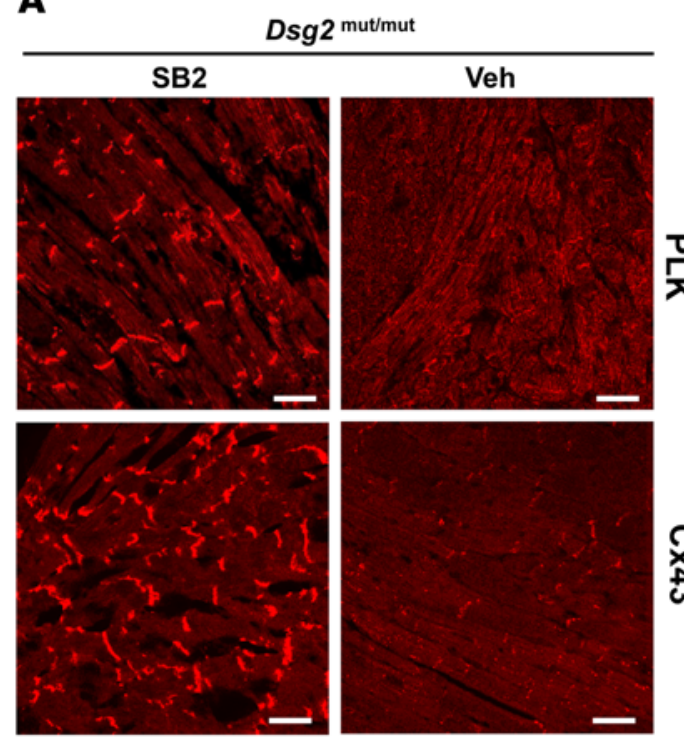

B
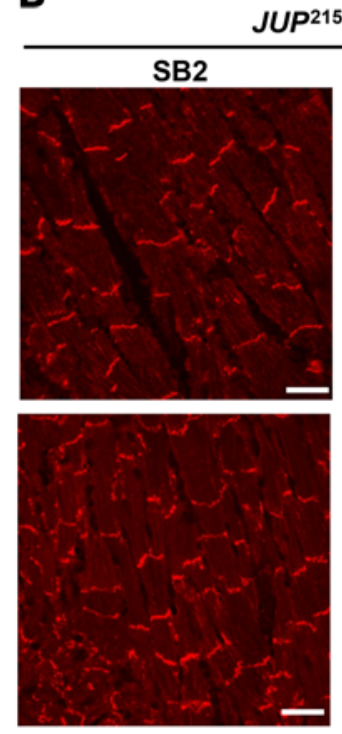

C

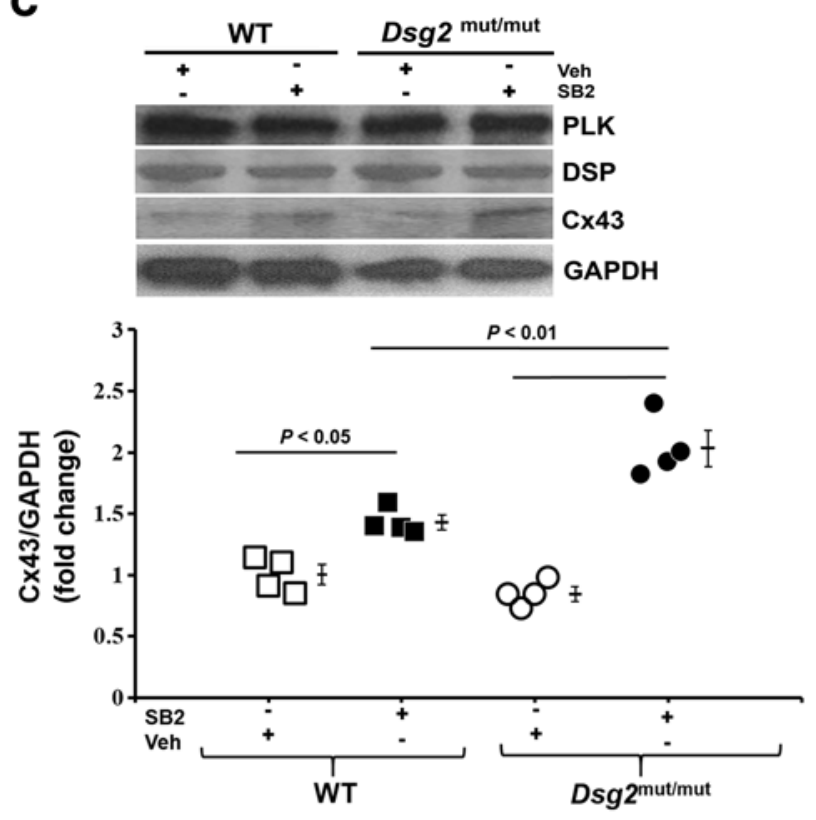

D
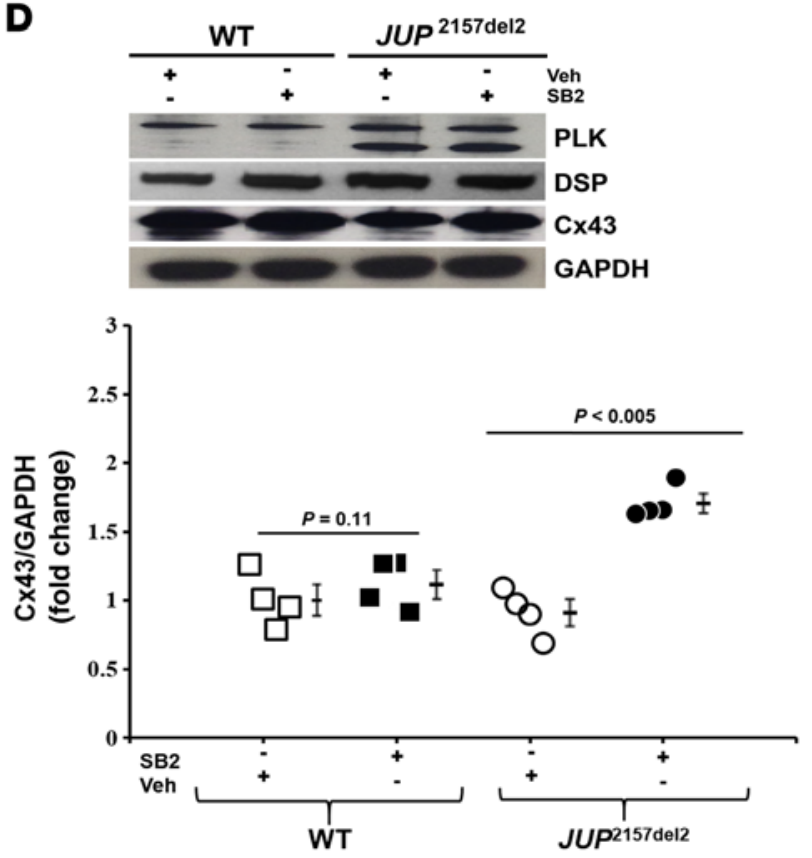

Figure 5. In vivo reversal of protein distribution in SB216763-treated ACM mice. (A and B) Representative myocardium immunolabeled with plakoglobin (PLK) and connexin43 (Cx43) from vehicle- (Veh-) and SB216763-treated (SB2-treated) WT, Dsg2 mut/mut, and JUP2157del2 mice. Scale bar: $20 \mu \mathrm{m}$. Images are representative of $n=4$ /genotype/treatment. (C and D) Western analysis of ventricular lysates probed for PLK, desmoplakin (DSP), and CX43, normalized to GAPDH. Mean \pm SEM, $n=4$ /genotype/treatment. $P<0.05$ for SB2-treated mice vs. Veh-treated mice using 2 -tailed paired $t$ test.

normalized by treatment with SB2 (Supplemental Figure 4). In both in vitro and in vivo models of ACM, GSK3 $\beta$ distribution was normalized after treatment with SB2 (Figure 6A). SB2 decreased total cellular protein levels of GSK3 $\beta$ in ACM mice (Figure 6, B and C), without an apparent change in phosphorylated GSK3 $\beta$ protein levels. Furthermore, regardless of vehicle or SB2 treatment, both mutant mouse models displayed reduced GSK3 $\alpha$ protein levels compared with WT controls (Figure 6, B and C). These findings suggest that SB2 specifically targeted the $\beta$ isoform of GSK3 (GSK3 $\beta$ ) and that the improvement in phenotypic manifestations in both murine models of ACM in response to SB2 is due to its effect on GSK3 $\beta$.

Considering GSK3 $\beta$ distribution was abnormally located at myocyte IDs in murine and NRVM mod- 
A

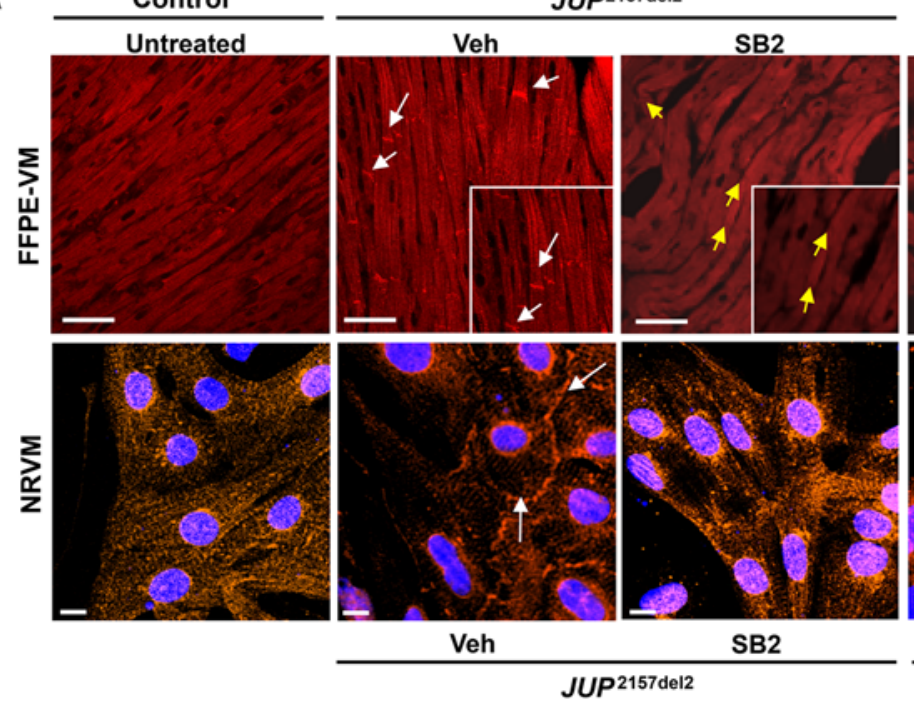

B

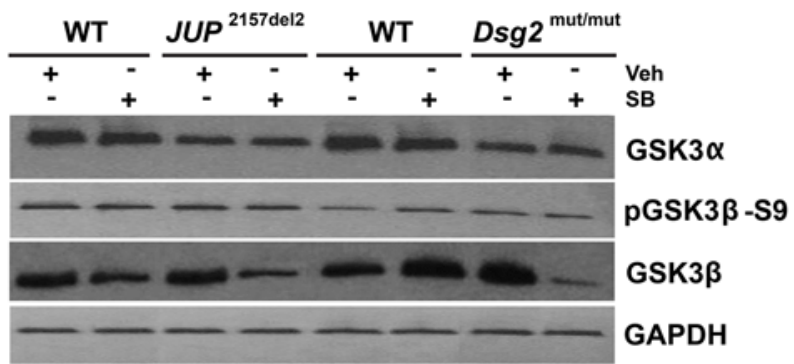

C

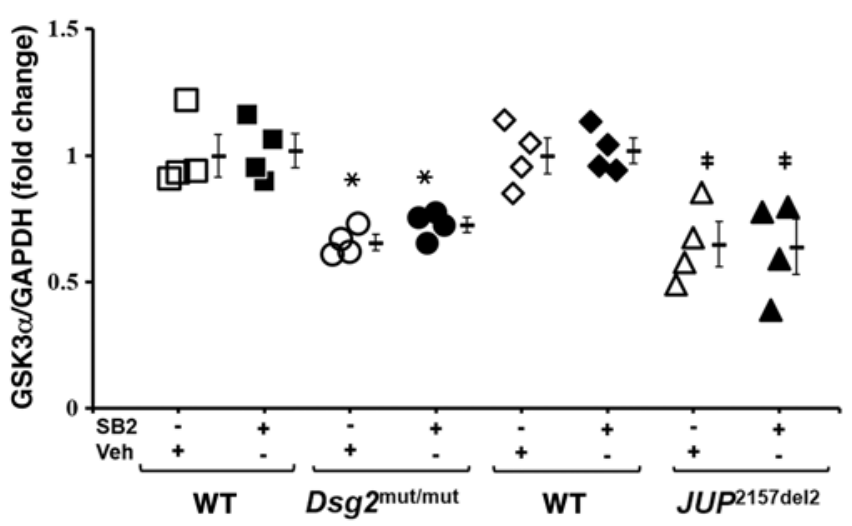

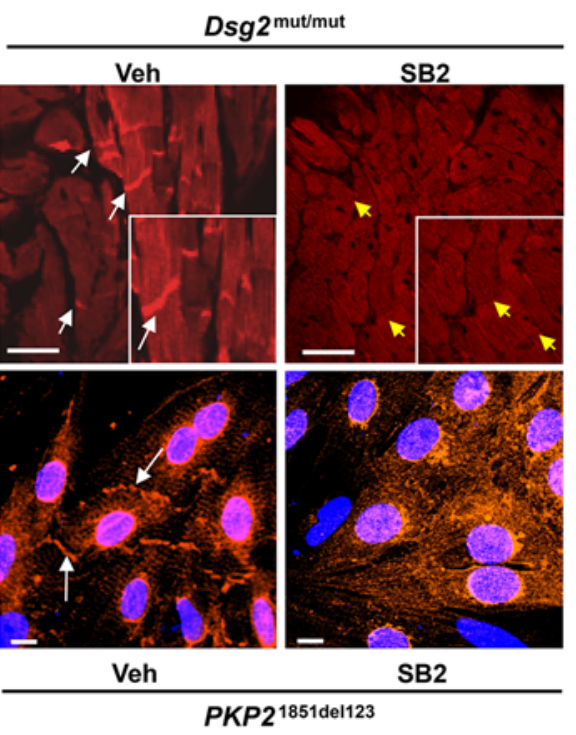

PKP2 1851del123

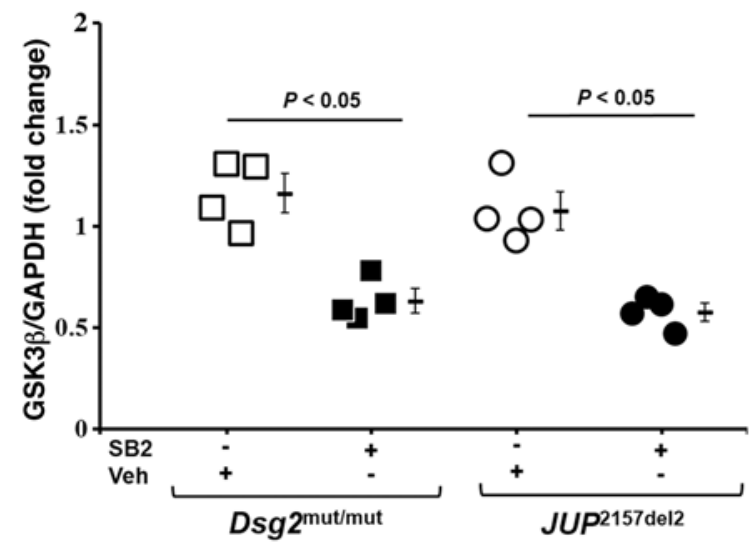

D

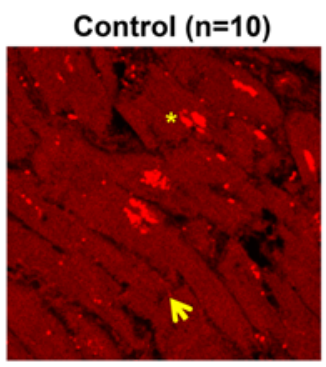

ACM \#1

$(n=20)$

ACM \#2
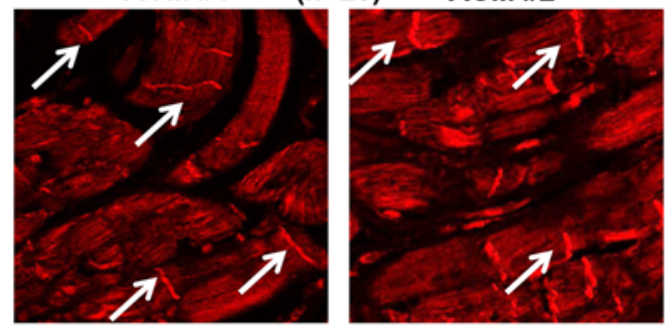

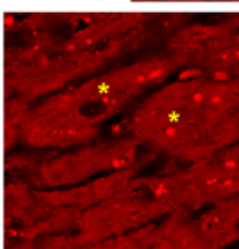

Ischemic $(n=5)$

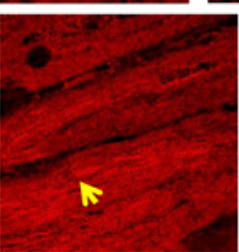

$\operatorname{DCM}(n=5)$

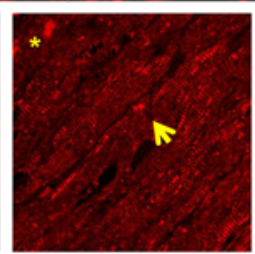

$\operatorname{HCM}(n=5)$

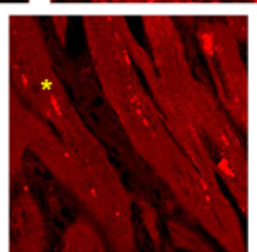

Sarcoidosis $(n=15)$

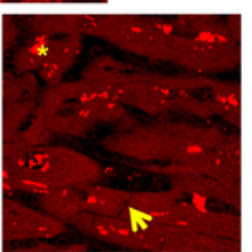

GCM $(n=5)$ 
Figure 6. GSK3 $\beta$ localization is uniquely abnormal in ACM, and SB216763 normalizes it. (A) Representative images of formalin-fixed, paraffin-embedded ventricular myocardia (FFPE-VM) (scale bar: $20 \mu \mathrm{m}$ ) from JUP2157del2 and Dsg2mut/mut mice and neonatal rat ventricular myocytes (NRVM) (scale bar: $10 \mu \mathrm{m}$ ) expressing JUP ${ }^{2157 d e 12}$ and $P K P 2^{1851 d \text { di123 }}$ transgenes immunolabeled for anti-GSK3 $\beta$. Images are representative of $n=4 /$ genotype/treatment (DAPI, blue; CSK3 $\beta$, red; white arrows, ID localization of GSK3 $\beta$; yellow arrows, absence of ID localization of GSK3 $\beta$ ). (B) Western blots probed for GSK3 $\alpha$, GSK3 $\beta$, and phosphorylated GSK3 $\beta$ (pGSK3 $\beta$-S9) from WT, Dsg2 $2^{\text {mut/mut }}$, and JUP2157del2 mice. (C) Quantitative GSK3 $\beta$ and GSK3 $\alpha$ protein levels from WT, Dsg2 ${ }^{\text {mut/mut, and }}$ JUP2157del2 mice, normalized to GAPDH. Mean \pm SEM, $n=4$ /genotype/treatment. $P<0.05$ for SB2-treated mice vs. Veh-treated mice using 2-tailed paired $t$ test. ${ }^{*} D s g 2^{\text {mut/mut }}$ vs. WT; ${ }^{\ddagger} J U P^{2157 d e l 2}$ vs. WT. (D) GSK3 $\beta$-immunolabeled patient biopsies. Representative images taken from endomyocardial biopsy samples show CSK3 $\beta$ distribution at IDs (white arrows) in all patients with ACM (20 of 20) differ from control hearts $(n=10)$ and patients diagnosed with sarcoidosis $(n=15)$, giant cell myocarditis (GCM, $n=5)$, and end-stage ischemic, dilated cardiomyopathy (DCM), and hypertrophic cardiomyopathy (HCM, $n=5$ for each) (yellow asterisks, punctate cytosolic pools of GSK3 $\beta$; yellow arrows, absence of GSK3 $\beta$ signal at IDs).

els of ACM, we investigated GSK3 $\beta$ localization in the hearts of patients with ACM and desmosomal mutations. We immunostained myocardia from patients bearing mutations in each of the major cardiac desmosomal genes implicated in ACM (JUP, DSP, PKP2, DSG2, and DSC2, $n=14)$ and from patients who met task force criteria (16) for the disease but in whom no desmosomal gene mutation had been identified $(n=6)$. For controls, 10 samples were obtained at autopsy from individuals with no clinical or pathological evidence of heart disease. Myocardia from patients with ACM showed abnormal GSK3 $\beta$ immunoreactive signal at myocyte IDs in all (20 of 20$)$ patients with ACM (Figure 6D), in contrast with normal control hearts $(n=10)$ and samples from patients with giant cell myocarditis $(n=5)$ and end-stage ischemic, dilated or hypertrophic cardiomyopathies ( $n=5$ for each). Although abnormal PKG immunostaining is also somewhat specific for ACM, hearts from patients with cardiac sarcoidosis mimic ACM (17). However, hearts from patients with cardiac sarcoidosis $(n=15)$ displayed a normal pattern of diffuse cytoplasmic localization and/or punctate bodies of GSK3 $\beta$ without accumulation of GSK3 $\beta$ at IDs (Figure 6D).

$S B 2$ treatment improves response to exercise in Dsg2 $2^{\text {mut }}$ mut mice. Exercise worsens disease penetrance and survival in animal models $(12)$ of ACM and in patients with $\mathrm{ACM}(18,19)$, so we reasoned that SB2 would improve cardiac response to exercise in ACM mice. $D s g 2^{\text {mut } / \mathrm{mut}}, D s g 2^{\mathrm{mut} /+}$, and WT mice were trained to swim at 5 weeks of age ( 10 minutes per day increments) and, by 7 weeks of age, mice swam 90 minutes per day, 5 days per week, until the endpoint - 16 weeks of age (Supplemental Figure 5A). Cardiac function was assessed by echocardiography at 16 weeks, after which ECG telemetry probes were implanted. Following 3 days of recovery, mice swam while ECG telemetry was recorded for 90 minutes. Preliminary exercise experiments (no vehicle or SB2 treatment, baseline exercise) were performed to assess percent survival and exercised-induced changes in cardiac function in heterozygous $D s g 2^{\text {mut } /+}$ mice.

In baseline exercise studies, survival was similar in WT and $D_{s g} 2^{\text {mut/ } /+}$ mice (Supplemental Figure 5B). In contrast, 6 of $11(55 \%) D s g 2^{\text {mut } / m u t}$ mice died while swimming, with mean time of exercise at death of $7.8 \pm 0.6$ weeks ( $P=0.029$ for log-rank survival test compared with WT mice; Supplemental Figure $5 \mathrm{~B})$. Exercised $D s g 2^{\mathrm{mut} / \mathrm{mut}}$ mice showed longer mean QRS complex duration and reduced S wave amplitude compared with $D s g 2^{\text {mut/ }}$ and WT mice (Supplemental Figure 5C). In contrast to sedentary $D s g 2^{\text {mut }}$ mut mice, exercised $D s g 2^{\text {mut }}$ mut mice displayed numerous TUNEL-positive nuclei (Supplemental Figure 6A). Exercised $D s g 2^{\mathrm{mut} / \mathrm{mut}}$ mice showed extensive biventricular fibrosis and inflammation, while $D_{s g} 2^{\mathrm{mut} /+}$ mice showed mild anteroapical and right ventricular fibrosis and inflammation (Supplemental Figure 6B).

SB2 improved survival in exercised $D s g 2^{\text {mut } / \text { mut }}$ mice, compared with exercised, vehicle-treated $D s g 2^{\text {mut }}$ mut mice (Figure 7A). Dsg2 $2^{\text {mut } / m u t}$ mice treated with vehicle had a median survival time of 8.5 weeks of age, with $50 \%$ (5 of 10) surviving to 16 weeks of age, whereas $D s g 2^{\text {mut/mut }}$ mice treated with SB2 had a median survival time of 13.5 weeks of age, with $70 \%$ (7 of 10) surviving to endpoint. SB2 improved LV function in $D s g 2^{\text {mut/mut }}$ mice at 16 weeks after exercise (Figure 7B). Exercised, vehicle-treated $D s g 2^{\text {mut } / \text { mut }}$ mice show reduced EF and FS compared with exercised, vehicle-treated $D_{s g} 2^{\mathrm{mut} /+}$ and WT mice (Supplemental Table 2). Exercised, vehicle-treated $D_{s g} 2^{\text {mut }}$ mut mice showed numerous TUNEL-positive nuclei with an increased apoptotic index compared with all swimmers (Figure 7C). All vehicle-treated swimmers (mutants and WT mice) exhibited some apoptotic cells. However, heterozygous and homozygous Dsg2 mutant mice exhibited higher numbers of TUNEL-positive nuclei than WT mice (Figure 7C). No mouse that received SB2 (regardless of the genotype) displayed any discernible apoptotic nuclei (Figure 7C).

Vehicle-treated, exercised $D s g 2^{\text {mut/mut }}$ mice demonstrated strong GSK3 $\beta$ signal at IDs, while $D s g 2^{\text {mut/ }}$ mice exhibited a heterogeneous pattern of GSK3 $\beta$ immunolabeled myocardium, between diffuse cytoplasmic and ID localization (Figure 7D). SB2-treated mutants normalized GSK3 $\beta$ distribution to control-like cytoplasmic distribution (Figure 7D). Both exercised mutant and WT mice exhibited control-like distribution of N-cad- 


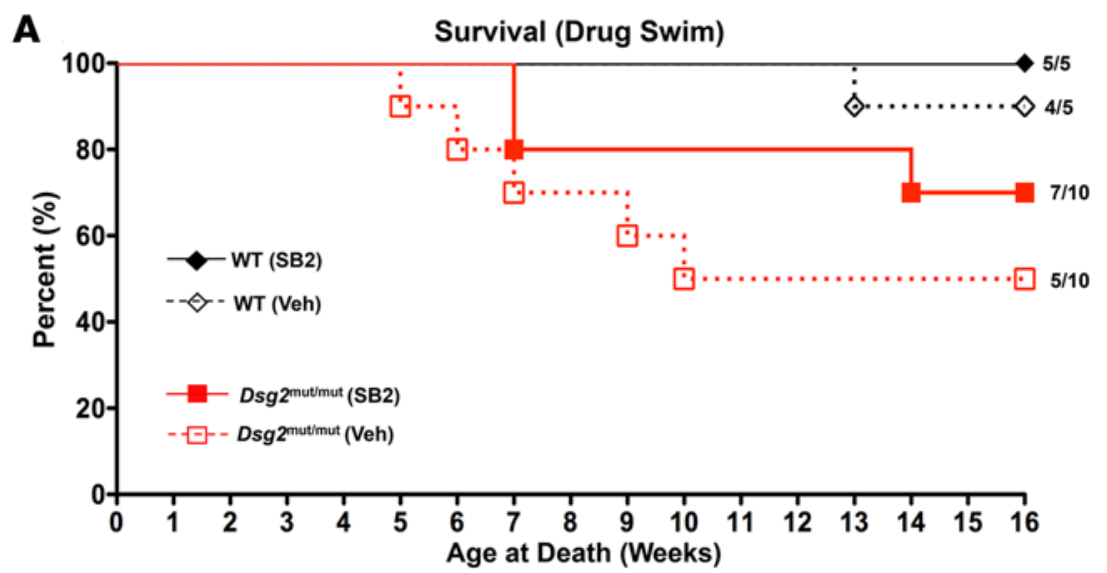

B
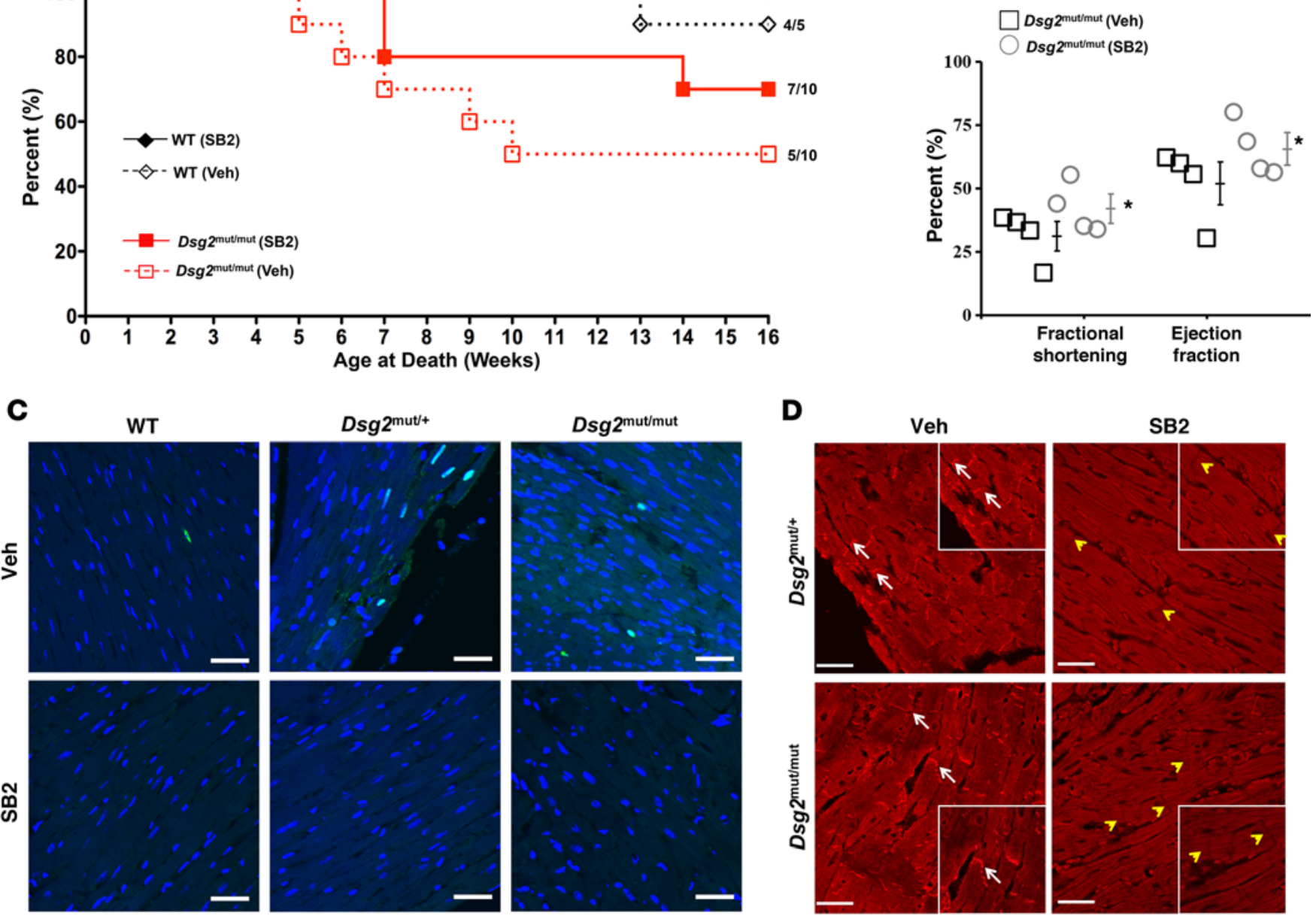

Dsg2 $2^{\text {mut/+ }}$

$D s g 2^{\text {mut/mut }}$

D
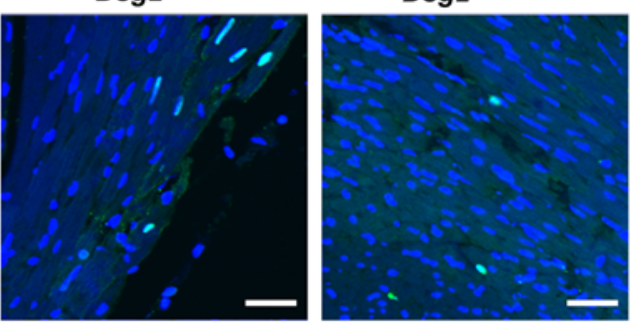

D

Veh

SB2
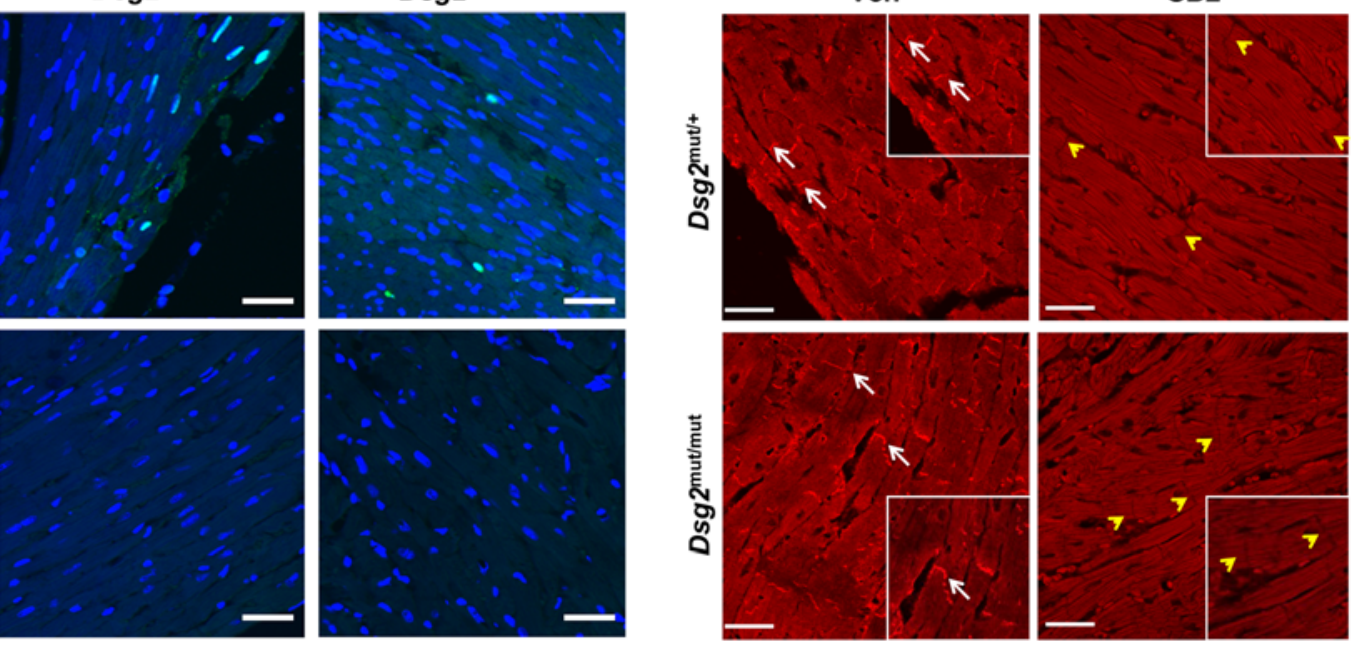

E

Dsg2 $2^{\text {mut/mut }}$

$D s g 2^{m u t /+}$

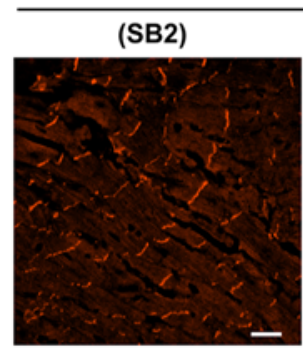

(Veh)

(SB2)
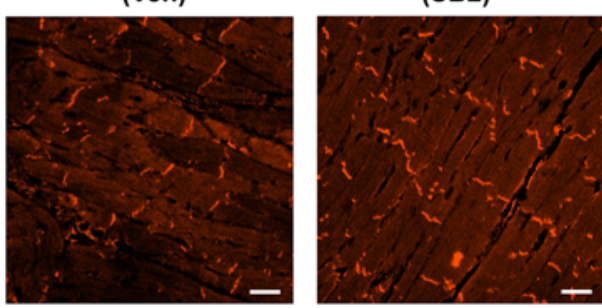

(Veh)
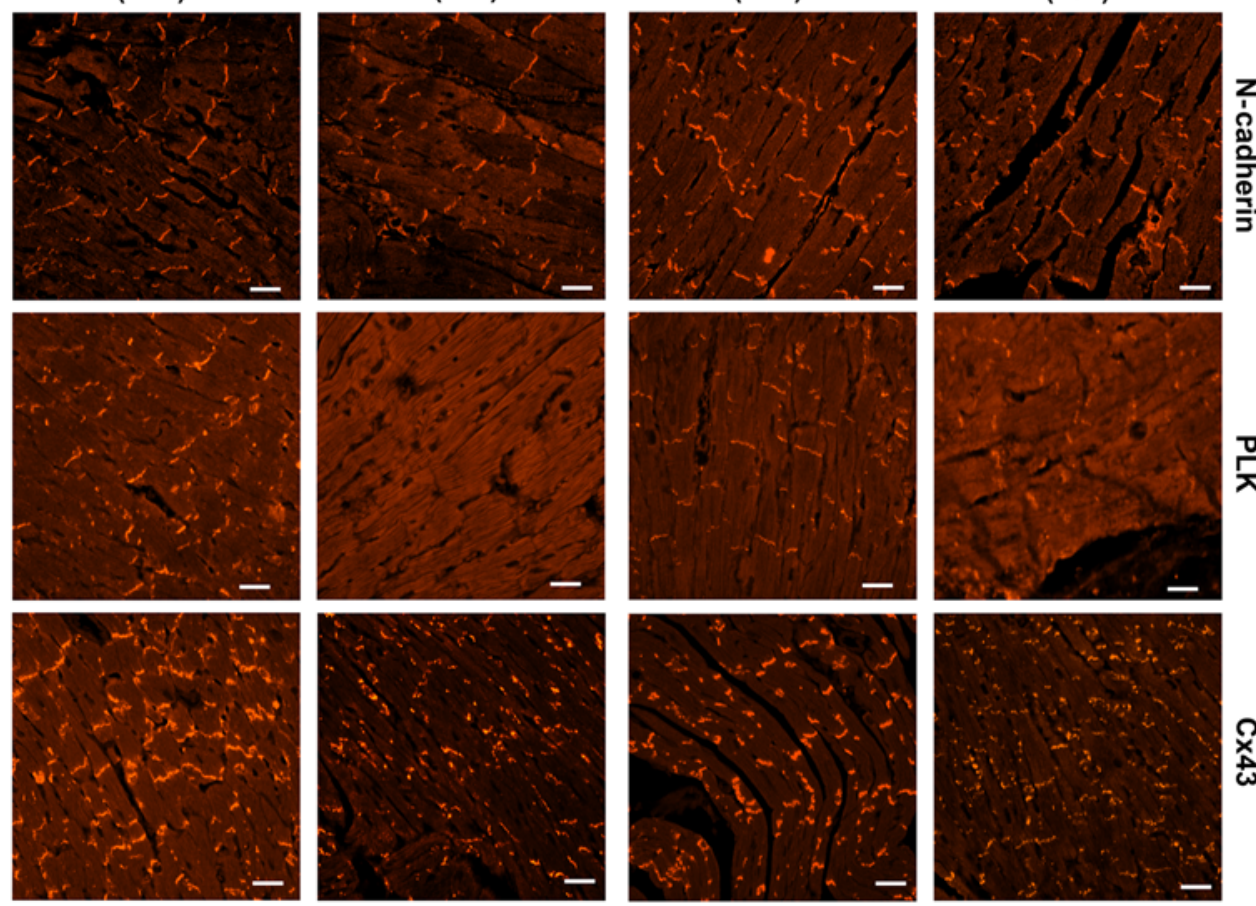
Figure 7. SB216763 treatment improves response to exercise in Dsg2 ${ }^{\mathrm{mut} / \mathrm{mut}}$ mice. (A) Percentage survival of drug-treated exercised Dsg2 ${ }^{\mathrm{mu} / \mathrm{mut}}$ and WT mice. Mean \pm SEM, $n$ values are shown. (B) Percentage ejection fraction and fractional shortening from exercised vehicle- (Veh-) and SB216763-treated (SB2-treated) $D s g 2^{\text {mut/mut }}$ mice at 16 weeks of age. Mean \pm SEM, $n=4 /$ genotype/treatment. ${ }^{*} P<0.05$ using 1-way ANOVA. (C) Myocardia immunolabeled with TUNEL from exercised Dsg2 mutant and WT mice treated with Veh or SB2. Scale bar: $20 \mu \mathrm{m}$. Images are representative of $n=4 / g e n o t y p e /$ treatment. (D) GSK3 $\beta$ immunolabeled myocardium. Images are representative of $n=4 /$ genotype/treatment (white arrows, junctional signal for GSK3 $\beta$; yellow arrowheads, absence of junctional signal for GSK3 $\beta$ ). Scale bar: $20 \mu \mathrm{m}$. (E) Representative images from exercised drug-treated Dsg2 ${ }^{\mathrm{mut} / \mathrm{mut}}$ and $D s g 2^{\mathrm{mut} /+}$ mice immunostained for plakoglobin (PLK) and connexin43 (Cx43). Images are representative of $n=4 /$ genotype/treatment. Scale bar: $20 \mu \mathrm{m}$.

herin, as assessed via immunofluorescence, regardless of treatment (Figure 7E). Immunolabeled myocardial samples from exercised, vehicle-treated $D s g 2^{\mathrm{mut} / \mathrm{mut}}$ and $D s g 2^{\mathrm{mut} /+}$ mice displayed abnormal distribution of PLK and $\mathrm{Cx} 43$, which were normalized in exercised mutant mice treated with SB2 (Figure 7E).

GSK $3 \beta$ knockdown prevents loss of junctional signal of ID proteins in ACM NRVMs. To determine whether the beneficial effects of GSK3 $\beta$ inhibition by SB2 were solely due to silencing of this kinase, we acquired an adenoviral construct expressing GSK $3 \beta$ shRNA that achieved $\geq 80 \%$ knockdown of total GSK $3 \beta$ protein, with no apparent change in GSK $\alpha$ protein levels, confirming $\beta$-isoform-directed specificity of viral construct (Figure 8A). Transfection with GSK3 $\beta$ shRNA had no effect on the distribution of PLK or Cx43 compared with untransfected control NRVMs (Figure 8B). Conversely, NRVMs transfected with mutant $P K P 2^{1851 \text { del123 }}$ showed increased nuclear PLK signal, with reduced junctional signal for PLK and Cx43 (Figure 8C). Naxos-transfected NRVMs ( $J U P^{2157 d e l 2}$ ) displayed markedly reduced junctional signal for PLK and Cx43, with abundant nuclear PLK localization compared with $P K P 2^{1851 \text { del123 }}$-transfected NRVMs (Figure $8 C)$. Next, we sought to determine whether GSK3 $\beta$ shRNA NRVMs would retain normal junctional signal for PLK and CX43 when transfected with either $P K P 2^{1851 \text { del123 }}$ or the $J U P^{2157 \text { del2 }}$ construct. Indeed, abnormal PLK and Cx43 distribution was prevented in GSK3 $\beta$ knockdown NRVMs when transfected with viruses delivering either $J U P^{2157 d \text { del2 }}$ or $P K P 2^{1851 \text { del123 }}$ constructs (Figure $8 \mathrm{C}$ ). Knockdown of GSK3 $\beta$ expression in transfected NRVMs was confirmed via Western immunoblotting (Figure 8A). Furthermore, GSK3 $\beta$ knockdown NRVMs transfected with ACM expressing constructs showed no effect on PLK or GSK3 $\alpha$ expression, with increased Cx43 expression levels (Figure 8A).

Dsg2 mutant mice with constitutively active GSK3 $\beta$ demonstrate increased myocardial fibrosis and cardiac dysfunction. Active GSK3 $\beta$ (nonphosphorylated Ser9) appears to protect the physiologically healthy heart by reducing the expression of profibrotic genes (6). Alternatively, GSK3 $\beta$ inactivation by phosphorylation at Ser9 is cardioprotective during ischemic injury to the heart (20). Therefore, we reasoned that one or more copies of a constitutively active form of GSK3 $\beta$ ( GSK3 $\beta$-S9A mutation, encoded kinase cannot be inactivated by phosphorylation at amino acid residue 9) would worsen cardiac function, histology, and arrhythmia in the context of a desmosome mutation. We bred a previously characterized strain of mice (21) with a knockin mutation in the murine Gsk3b gene (Ser9 was mutated to alanine) with our Dsg2 mutant line. Homozygous $D s g 2$ mutant mice with 1 or 2 copies of the constitutively active GSK3 $\beta$ mutant allele (Dsg2 $2^{\mathrm{mut} / \mathrm{mut}} ; G S K 3 \beta^{\mathrm{s9A} /+}$ or $D s g 2^{\mathrm{mut} / \mathrm{mut}} ; G S K 3 \beta^{\mathrm{s9A} / \mathrm{s9A}}$ ) exhibited reduced cardiac function by 8 and 16 weeks of age compared with WT controls (Figure 9, A and B), as assessed by echocardiography.

$D s g 2^{\mathrm{mut} /+}$ mice displayed normal cardiac function and histology at rest, yet developed disease phenotypes in response to exercise (Supplemental Figure 5 and Figure 7). Therefore, we sought to determine whether $D s g 2^{\mathrm{mut} /+}$ mice bearing 1 or 2 copies of the constitutively active GSK3 $\beta$ mutant allele (Dsg2 ${ }^{\mathrm{mut} /+} ; G S K 3 \beta^{S 9 \mathrm{~A} /+}$ or $D s g 2^{\mathrm{mut} /+} ; G S K 3 \beta^{\mathrm{S9A} / \mathrm{S9A}}$ ) would display disease characteristics compared with WT controls. Dsg2 ${ }^{\mathrm{mut} /+}$ mice exhibited nearly normal cardiac function (Table 1), regardless of the presence of 1 or 2 copies of mutant GSK3 $\beta$-S9A at 16 weeks of age, although more subtle ECG abnormalities were present in these mice (Supplemental Table 3 and Supplemental Figure 7, A and B). Similar ECG abnormalities were present in the $D s g 2^{\mathrm{mut} / \mathrm{mut}}$ mice with 1 or 2 copies of the constitutively active GSK3 $\beta$-S9A allele (Supplemental Table 3 and Supplemental Figure 7, A and B). Prolongation of the PR interval was observed in Dsg2 mutant mice with 1 or 2 copies of the constitutively active $G S K 3 \beta-S 9 A$ allele as well as in homozygous $G S K 3 \beta-S 9 A$ mice ( $G S K 3 \beta^{59 \mathrm{~A} / \mathrm{s9} \mathrm{A}}$, without $D s g 2$ mutation), reflecting a known role of canonical Wnt signaling in regulation of $\mathrm{AV}$ junction electrophysiology (22). Although GSK3 $3 \beta^{\mathrm{S9A} / \mathrm{S9A}}$ mice exhibited prolonged PR interval, GSK3/ $\beta^{\text {S9A } / 59 \mathrm{~A}}$ mice displayed normal QRS duration and S Amplitude values (Supplemental Table 3 and Supplemental Figure 7, A and B) compared with Dsg2 mutant mice with 1 or 2 copies of constitutively active GSK3B-S9A allele. Furthermore, immunohistochemical analysis in myocardia from $D s g 2^{\mathrm{mut} / \mathrm{mut}}$ mice bearing 1 or 2 copies of mutant GSK3B-S9A alleles showed extensive biventricular fibrosis (Figure 9C) compared with WT controls. 
A

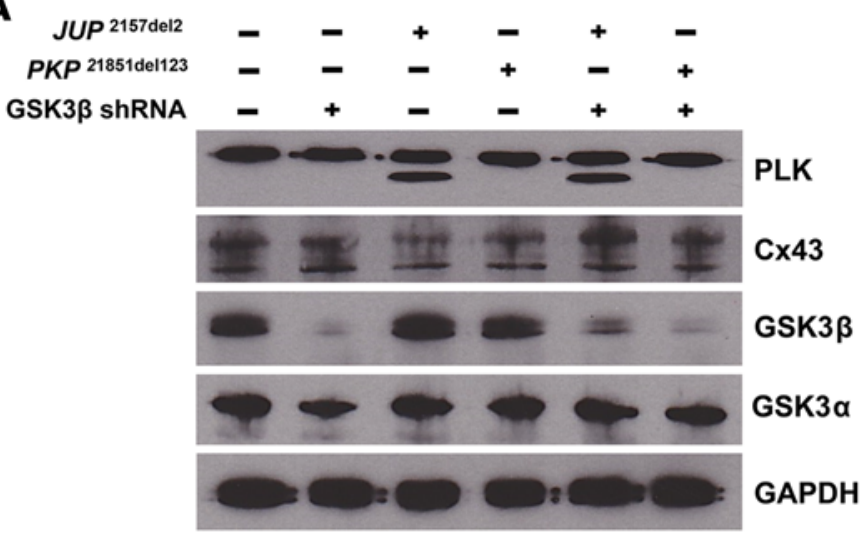

B
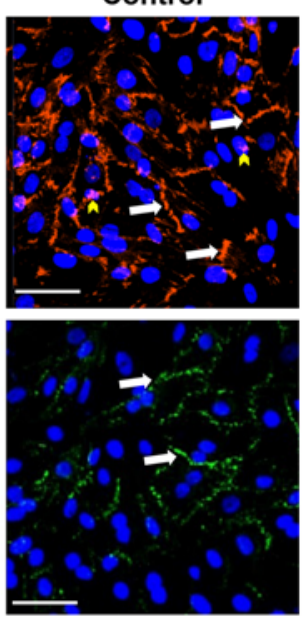

GSK3ß-shRNA
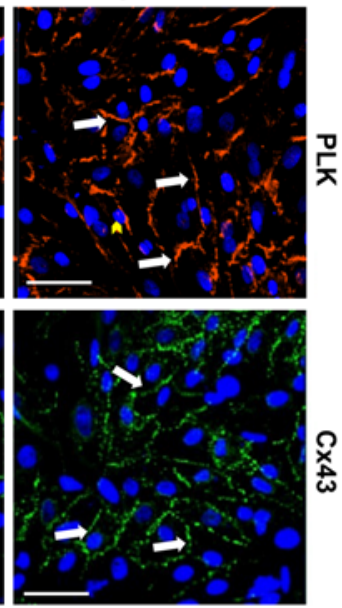

C
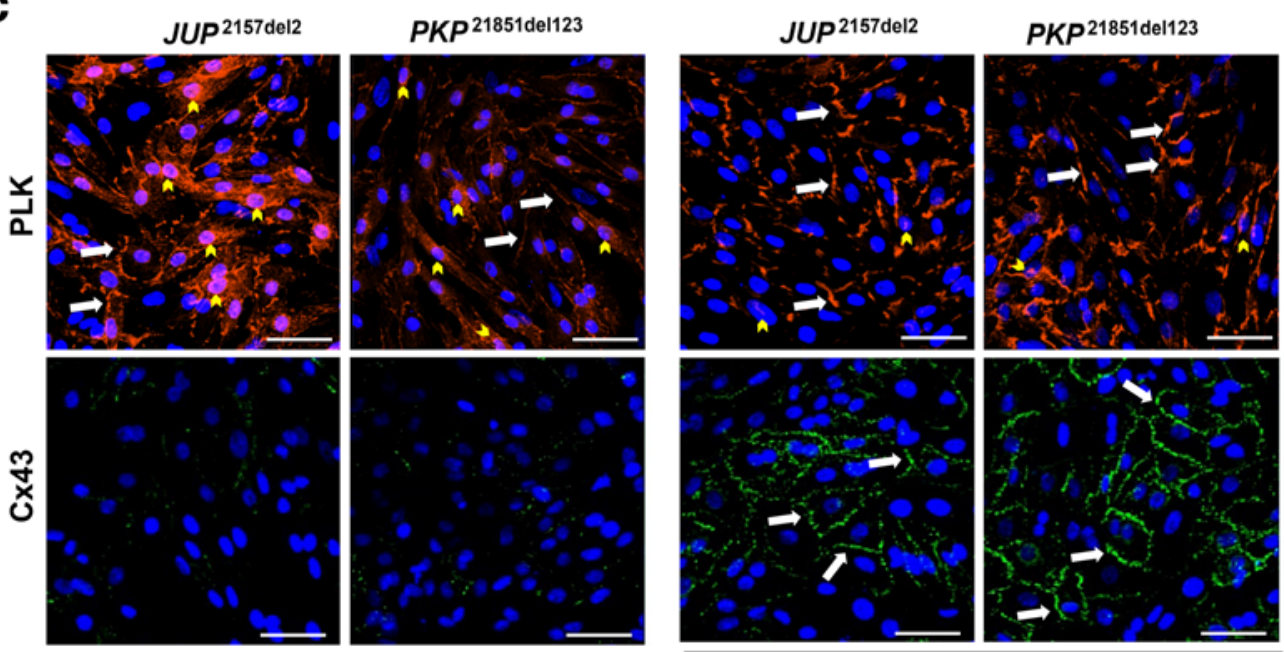

GSK3ß-shRNA

Figure 8. GSK3 $\beta$ knockdown prevents loss of junctional signal of ID proteins in ACM neonatal rat ventricular myocytes. (A) GSK3 $\beta$ knockdown was confirmed via Western immunoblots in CSK3 $\beta$ shRNA-transfected neonatal rat ventricular myocytes (NRVMs) compared with untransfected controls. Cotransfected NRVM lysates probed for changes in plakoglobin (PLK), connexin43 ( $C \times 43$ ), and GSK3 $\alpha / \beta$, normalized to GAPDH. Immunoblots are representative of $n=3 /$ cohort. (B) Representative images of untransfected controls and GSK3 $\beta$ shRNA-transfected NRVMs. Note that GSK3 $\beta$ shRNA-transfected NRVMs display control-like PLK and Cx43 distribution. Images are representative of $n=3 /$ cohort. (C) Mutant ACM expressing NRVMs displayed increased nuclear PLK localization and near absent Cx43 signal. Cotransfected NRVMs (ACM expressing construct and GSK3 $\beta$ shRNA transgene) immunostained for changes in PLK and Cx43 distribution. Note that abnormal PLK and Cx43 localization was prevented in cotransfected NRVMs, and Cx43 signal was increased compared with ACM myocytes without CSK3 $\beta$ shRNA construct (white arrows, junctional signal; yellow arrowheads, nuclear localization). Scale bar: $50 \mu \mathrm{m}$. Images are representative of $n=3 /$ cohort.

\section{Discussion}

Although the genetic basis of ACM is well recognized as a disorder of the cardiac desmosome, the pathogenesis of this inherited form of cardiomyopathy and sudden cardiac death has been elusive. Early evidence of altered canonical Wnt signaling invoked disruption of the desmosome complex, with $\gamma$-catenin (PLK) competitively inhibiting $\beta$-catenin signaling (2). However, this model does not adequately explain mutations in PLK, as seen in Naxos syndrome. By recapitulating ACM in two different murine models, one with transgenic overexpression of mutant JUP, encoding Naxos PLK, and the other with a knockin mutation in murine Dsg2, encoding desmoglein-2, our results showed common structural, histopathological, and arrhythmic consequences. All of these manifestations of ACM respond favorably to GSK3 $\beta$ inhibition and adversely to constitutive GSK3 $\beta$ activation.

The mutant Dsg2 model supports loss of function as a valid mechanism of disease with desmoglein-2 mutations. Although previous studies demonstrated that Dsg2-null mice are embryonic lethal (23), this new 
A

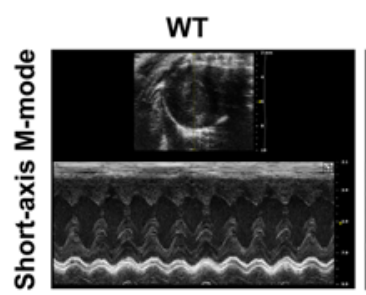

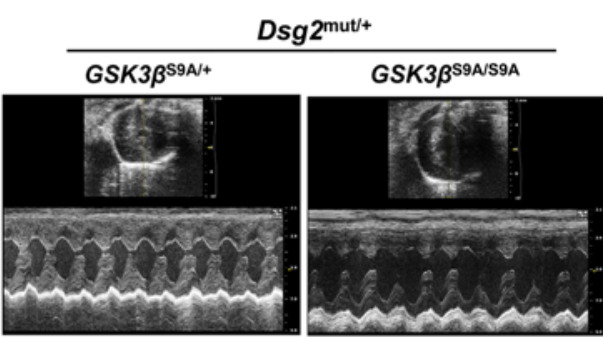

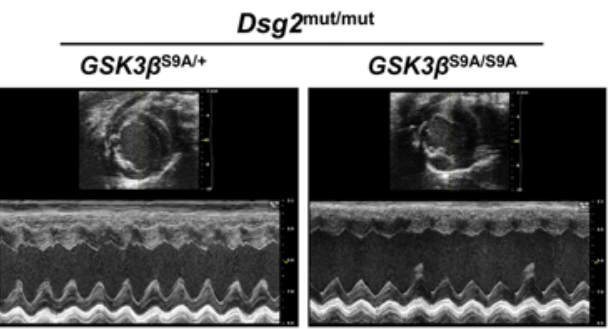

B

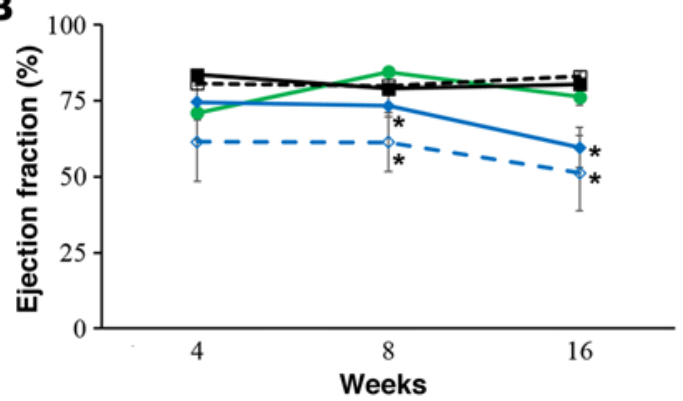

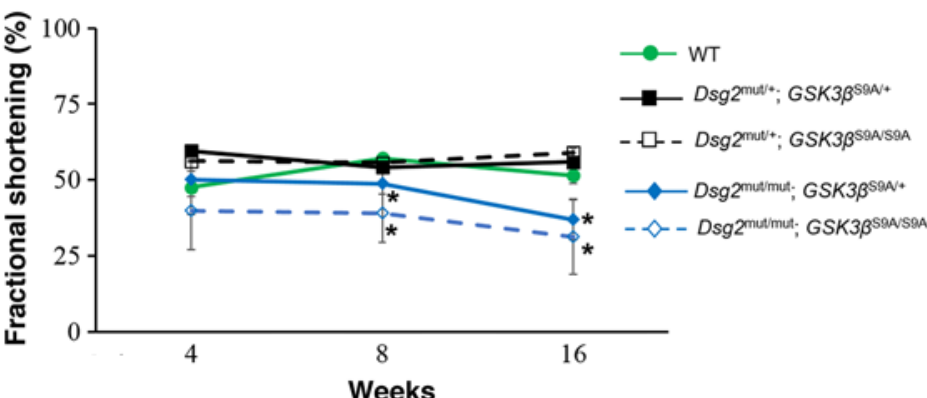

Weeks

C

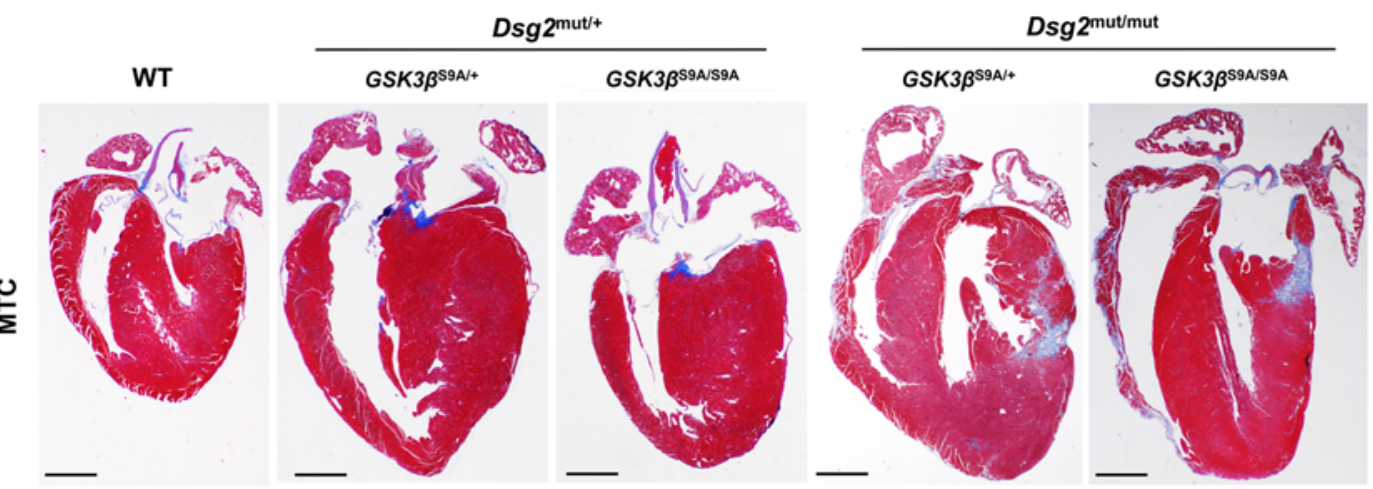

Figure 9. Dsg2 mutant mice with constitutively active GSK3 $\beta$ demonstrate increased myocardial fibrosis and cardiac dysfunction. (A) Representative short-axis, m-mode echocardiography from WT and heterozygous and homozygous Dsg2 mutant mice with 1 or 2 copies of mutant CSK3 $\beta$-S9A alleles. Images are representative of $n \geq 4 /$ genotype. (B) Quantitative echocardiography analysis of WT and heterozygous and homozygous Dsg2 mutant mice

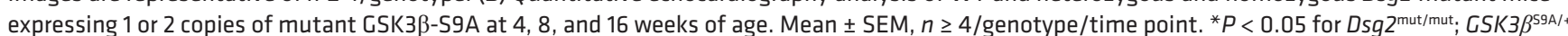
and Dsg2 ${ }^{\text {mut/mut; }}$ CSK3 $\beta^{59 A / 59 A}$ vs. WT using 2-way ANOVA with Tukey's post-hoc analysis. (C) Representative images of ventricular myocardia from WT, heterozygous, and homozygous Dsg2 mutant mice with either 1 or 2 copies of mutant GSK3ß-S9A stained with Masson's trichrome (MTC). Images are representative of $n=4 /$ genotype. Scale bar: $1 \mathrm{~mm}$.

strain of homozygous Dsg2 mutant mice do indeed survive into adulthood. We chose not to create a cardiac-specific mutation in order to mimic human ACM more closely (11). Hypomorphic expression of the targeted Dsg2 allele, prior to recombination and deletion of 2 exons, causes functional and histologic features of ACM. Similarly, Krusche et al. developed a strain of mice with in-frame deletion of exons 4-6 of $D s g 2$, and they concluded that loss of function rather than dominant-negative interference was responsible for the observed cardiomyopathy in these mice (24). Notably, two-thirds of the homozygous Dsg2 mutant mice with targeted deletion of exons 4-6 died in utero (24).

In the heterozygous $J U P^{2157 \mathrm{del} 2}$ murine model of ACM, the transgene is expressed at similar levels to endogenous Jup, implicating dominant-negative effects of the transgenic mutant protein as the cause of ACM phenotypes in these mice. This parallels similar findings from previous studies that demonstrated that dominant-negative interference of a mutant $J U P$ transgene recapitulates ACM disease phenotypes in mice and zebrafish $(3,25)$. Conversely, Zhang et al. showed that homozygous and heterozygous Jup mutant mice, expressing the original Naxos (OriNax) truncated Jup transgene, did not develop ACM disease phenotypes (26). However, in the study by Zhang et al., homozygous OriNax mice expressed significantly lower levels of only the exogenous truncated JUP protein, with a complete absence of endogenous JUP protein, and had a 
Table 1. Echocardiographic and morphometric indices from 16-week-old WT, GSK3 $\beta^{59 A / 59 A}$, and Dsg2 mutant mice with 1 or 2 copies of constitutively active GSK3ß-S9A

\begin{tabular}{|c|c|c|c|c|c|c|}
\hline & \multirow[t]{2}{*}{ WT } & \multirow[t]{2}{*}{ CSK $3 \beta^{59 A / S 9 A}$} & \multicolumn{2}{|c|}{$D s g 2^{m u t /+}$} & \multicolumn{2}{|c|}{ Dsg2 $2^{m u t / m u t}$} \\
\hline & & & CSK3 $3 \beta^{59 A /+}$ & $C S K 3 \beta^{59 A / S 9 A}$ & CSK3 $3 \beta^{59 A /+}$ & $C S K 3 \beta^{59 A / S 9 A}$ \\
\hline \multicolumn{7}{|l|}{ Echocardiographic } \\
\hline IVSd (mm) & $0.92 \pm 0.02$ & $0.96 \pm 0.03$ & $0.89 \pm 0.02$ & $0.94 \pm 0.02$ & $0.87 \pm 0.02^{A}$ & $0.80 \pm 0.07$ \\
\hline IVSs (mm) & $1.48 \pm 0.04^{A}$ & $1.57 \pm 0.03^{B}$ & $1.41 \pm 0.04^{\mathrm{A}}$ & $1.42 \pm 0.04^{A}$ & $1.27 \pm 0.06^{\mathrm{A}, \mathrm{B}}$ & $1.11 \pm 0.10^{A, B}$ \\
\hline LVIDd (mm) & $3.01 \pm 0.23$ & $2.75 \pm 0.16$ & $2.99 \pm 0.15$ & $3.02 \pm 0.14$ & $3.24 \pm 0.13^{A}$ & $3.27 \pm 0.26$ \\
\hline $\mathrm{EF}(\%)$ & $76.3 \pm 2.8$ & $82.1 \pm 0.5$ & $80.5 \pm 1.7$ & $81.0 \pm 2.6$ & $62.6 \pm 4.4^{\mathrm{A}, \mathrm{B}}$ & $54.5 \pm 6.8^{\mathrm{A}, \mathrm{B}}$ \\
\hline \multicolumn{7}{|l|}{ Morphometric } \\
\hline$n$ & 4 & 4 & 4 & 5 & 7 & 9 \\
\hline RWT (mm) & $0.99 \pm 0.06^{A}$ & $0.7 \pm 0.05^{\mathrm{B}}$ & $0.95 \pm 0.06^{A}$ & $0.86 \pm 0.09$ & $0.67 \pm 0.06^{\mathrm{B}}$ & $0.60 \pm 0.06^{\mathrm{B}}$ \\
\hline LVM (mg) & $12.8 \pm 2.8$ & $10.1 \pm 1.1$ & $11.5 \pm 1.9$ & $11.4 \pm 1.1$ & $12.6 \pm 1.1$ & $12.0 \pm 1.8$ \\
\hline
\end{tabular}

$100 \%$ PD1 lethality (26). Heterozygous OriNax mice expressed very low levels of the exogenous truncated JUP protein with control-like levels of endogenous JUP protein at PD1 (26). Although homozygous and heterozygous OriNax mice did not display characteristics of ACM, this may be due to the observed time point (PD1) in homozygous OriNax mice and the nonequivalent levels of exogenous truncated JUP protein compared to levels of endogenous JUP protein in heterozygous OriNax mice (26). To avoid the possibility of the abnormalities observed being due to disruption of unrelated genes, we created 4 founder lines (in which the exogenous gene was introduced at different areas of the genome and presumably at different copy numbers). The exogenous gene was expressed in all 4 lines (as shown by PCR reactions and Western immunoblotting), and all 4 lines showed similar abnormalities in terms of protein redistribution and histological and structural changes of the myocardium. This observation suggests that the changes observed are due to expression of the mutant PLK gene and not due to disruption of unrelated genes. We demonstrate here that $J U P^{2157 \mathrm{del} 2}$ mice have normal cardiac maturation from birth to 4 months of age, while ventricular arrhythmias and overt structural remodeling occur at 6 months of age due to dominant-negative interference of equivalent levels of exogenous truncated JUP protein compared with endogenous JUP protein.

Investigation of SB2, annotated as a GSK3 $\beta$ inhibitor $(4,5)$, has been crucial in demonstrating the potential role of aberrant GSK3 $\beta$ signaling in the pathogenesis of ACM. SB2 is an arylindolemaleimide, which works by occupying the ATP-binding site of GSK3 $\beta$, inhibiting the ability of GSK3 $\beta$ to phosphorylate target substrates $(4,5)$. This small molecule has been shown to prevent and/or reverse changes in the distribution of key ID proteins in ACM, including desmosomal proteins, connexins, and ion channels as well as SAP97, a protein that regulates expression of ion channels (Nav1.5, Kir2.1, Kv4.x) and PLK at the ID (27-30). Here, we show that the $\beta$ (not the $\alpha$ ) isoform of GSK3 appears to translocate from cytoplasmic to junctional pools in murine and NRVM models of ACM. Cell density, orientation, and confluence are essential for immunohistochemical studies involving the localization of ID proteins. As such, GSK3 $\beta$ localization at the ID was additionally confirmed in ACM mutant engineered NRVMs. Longitudinal paired myocytes fixed to fibronectin-plated glass slides demonstrated ID localization of GSK3 $\beta$ in mutant ACM paired myocytes sharing intact cell-cell junctions. Perhaps most importantly, abnormal ID localization of GSK3 $\beta$ was observed in myocardia from patients with ACM. Redistribution of GSK3 $\beta$ to cardiac IDs appears to be specific for ACM, as it was not evident in myocardia from patients with dilated, hypertrophic or ischemic cardiomyopathy, cardiac sarcoidosis, or giant cell myocarditis. Distribution of GSK3 $\beta$ is restored in all in vivo and in vitro models treated with SB2. Collectively, these data suggest a key role for 
aberrant GSK3 $\beta$ signaling in the final common disease pathway in ACM.

Prior work indicates that ACM is related to abnormal cell-cell mechanical coupling (31). This idea comes from EM studies showing gaps in IDs in ACM patients and mouse models (32). Knockdown of Pkp2 in NRVMs decreases cell-cell adhesion in vitro (measured by dispase assays) (33). Taken together, these findings support a primary structural deficiency, imposed by desmosome gene mutations, as an explanation of the pathogenesis of ACM. This model does not adequately explain fibrofatty scars in the heart or arrhythmias, which may occur early in the course of ACM. In contrast, abnormal GSK3 $\beta$ distribution arising from the structural consequences of a desmosome mutation do help to explain associated histologic, functional, and arrhythmic features of ACM. However, GSK3 $\beta$ is expressed ubiquitously throughout the heart, and myriad signaling pathways are regulated by this complex kinase. Therefore, caution should be implemented in the interpretation of our findings, as upstream signaling pathways were not extensively studied and may also contribute to disease development.

Exercise increases the incidence of ventricular arrhythmias and sudden death in patients bearing ACMcausing mutations, and it has been associated with accelerated disease progression $(18,19)$. Others have shown that exercise worsens disease phenotypes in mice with desmosome mutations $(12,34)$. Similarly, we showed that heterozygous $\mathrm{Dsg} 2^{\mathrm{mut} / \mathrm{+}}$ mice do not show an overt ACM phenotype under resting conditions but develop myocyte injury and redistribution of ID proteins in response to endurance exercise. Importantly, activation of the common disease pathway in $D s g 2^{\text {mut/t }}$ animals subjected to swimming was blocked by SB2. Treatment with SB2 also improved cardiac function, myocardial injury, and survival in exercised $D s g 2^{\mathrm{mut} / \mathrm{mut}}$ mice, implicating the same central disease pathway of aberrant GSK3 $\beta$ signaling in the pathogenesis of ACM progression in response to exercise.

A recent review indicated that nearly all pharmacological inhibitors of GSK3 available are not isoform specific and that use of these agents may result in nonspecific inhibition and off-target effects (6). However, we demonstrate here that SB2 indeed selectively targets the $\beta$-isoform of GSK3 in both ACM mutant mice, while expression of the $\alpha$-isoform of GSK3 is unaffected. Furthermore, selective GSK3 $\beta$ knockdown in NRVMs expressing desmosome mutations also prevents ACM disease phenotypes. Indeed, NRVMs expressing either a JUP mutation (2157del2) or a PKP2 mutation (1851del123) demonstrate normalized localization of ID proteins and increased $\mathrm{Cx} 43$ protein levels in response to prior GSK3 $\beta$ knockdown. Although these results indicate a critical role for GSK3 $\beta$ in the pathogenesis of ACM, adequate caution is required when interpreting the results, as other kinases may also be affected by this small molecule.

Previous studies report that active GSK3 $\beta$ reduces the expression of profibrotic genes in healthy hearts (6-10). In contrast, inhibition of this kinase by phosphorylation at Ser9 is required for cardioprotection in the pathologically ischemic heart (20). We hypothesized that disease manifestations would be worsened by the presence of one or more copies of a constitutively active form of GSK3 $\beta$ (GSK3 $\beta$-S9A knockin mutation) (21). A founder line of homozygous GSK3 $\beta^{\mathrm{s9A} / \mathrm{s} 9 \mathrm{~A}}$ mice (21) was bred with homozygous $D \operatorname{sg} 2^{\mathrm{mut} / \mathrm{mu}}$ mice, and double-heterozygote offspring (Dsg $\left.2^{\mathrm{mut} /+} ; G S K 3 \beta^{S 9 \mathrm{~A} /+}\right)$ were then crossbred with $D s g 2^{\mathrm{mut} /+}$; GSK $3 \beta^{\mathrm{S9A} /+}$ littermates. Offspring from double-heterozygote crossbreeds were then assessed for changes in cardiac function and myocardial injury at endpoint (16 weeks of age). Homozygous Dsg2 mutant mice bearing 1 or 2 constitutively active GSK3 $\beta$ alleles (Dsg2 $2^{\mathrm{mut} / \mathrm{mut}} ; G S K 3 \beta^{\mathrm{S9A} /+}$ and $D s g 2^{\mathrm{mut} / \mathrm{mut}} ; G S K 3 \beta^{\mathrm{S9A} / \mathrm{S9A}}$ ) demonstrated accelerated cardiac dysfunction and biventricular scarring compared with WT controls. Furthermore, Dsg2 $2^{\mathrm{mut} /+} ; G S K 3 \beta^{\mathrm{S9A} /+}, D s g 2^{\mathrm{mut} /+} ; G S K 3 \beta^{59 \mathrm{~A} / \mathrm{s} 9 \mathrm{~A}}, D s g 2^{\mathrm{mut} / \mathrm{mut}} ; G S K 3 \beta^{\mathrm{S9A} /+}$, and $D s g 2^{\mathrm{mut} / \mathrm{mut}}$; $G S K 3 \beta^{\mathrm{S9A} / \mathrm{S9A}}$ mice displayed prolonged QRS compared with GSK3$\beta^{\mathrm{S9A} / \mathrm{S9A}}$ and WT mice.

A recent review indicated that normal mouse ECGs differ from those obtained in healthy humans by the presence of a positive $J$ wave and a negative $T$ wave (35). In addition to increased QRS intervals, our double-mutant mice exhibited changes in the QRS morphology, such as an absent or negative J wave and a significant reduction in the S-amplitude. The reduction in S-amplitude in our double-mutant mice parallels similar ECG findings in mouse models of myocardial infarction (35). Specifically, Boukens et al. demonstrated that mouse models of ischemia (in the context of a myocardial infarction) present with absent or negative $\mathrm{J}$ waves and a significant reduction in the S-amplitude. Additionally, heterozygous $D s g 2^{\mathrm{mut} /+}$ mice bearing either 1 or 2 copies of constitutively active GSK3 $\beta$ exhibited control-like EF and FS values, yet prolonged QRS and PR intervals. GSK3 $\beta^{\mathrm{S9A} / \mathrm{S9A}}$ founder mice showed control-like echocardiographic, morphometric, and electrocardiographic parameters; albeit, they did exhibit one abnormal ECG manifestation compared with WT mice - PR prolongation. Importantly, the presence of the Dsg2 mutation (with no GSK3 $3 \beta^{59 \mathrm{~A}}$ mutation) did not worsen PR prolongation compared with WT and GSK3 $3 \beta^{\mathrm{S9A} / \mathrm{S9A}}$ mice. 
Therefore, we hypothesize PR prolongation in Dsg2 mutant mice with 1 or 2 copies of constitutively active GSK $3 \beta$ is simply due to the presence of mutant GSK3 $\beta$ allele.

We show here that pharmacological inhibition of GSK3 $\beta$ can prevent or delay disease phenotypes in two different cell and mouse models of ACM in vitro and in vivo, respectively. Patients with ACM have a unique pattern of GSK3 $\beta$ mislocalization at the myocyte ID on immunohistochemical staining compared with other forms of heart disease. Silencing of GSK3 $\beta$ improves features of ACM in NRVMs expressing either JUP or PKP2 mutations, and constitutive activation of GSK3 $\beta$ accelerates ACM pathology in Dsg2 mutant mice. Taken together, these findings indicate that a central disease pathway via aberrant GSK3 $\beta$ signaling contributes to the pathogenesis of ACM. Variation in this central disease pathway may help to explain the age-related progression, phenotypic variation, and reduced genetic penetrance characterizing ACM.

\section{Methods}

\section{Generation of experimental murine models of ACM}

Dsg $2^{\text {mut } / m u t}$ mutant mice. We obtained 3 targeted murine embryonic stem clones, containing an inducible knockin mutation in Dsg2 and a removable neomycin-resistance cassette, from the European Conditional Mouse Mutagenesis Program. We performed microinjection into murine blastocysts, developed 17 chimeric mice with moderate to high levels of coat color derived from the embryonic stem cell microinjection, and backcrossed the mice into $\mathrm{C} 57 \mathrm{BL} / 6 \mathrm{~J} \times>8$ generations after confirming germline incorporation of the correctly targeted Dsg2 gene. Mice with the targeted allele, without Cre-mediated loss of exons 4 and 5 or Flp-mediated removal of the neomycin-resistance cassette, were designated $D s g 2^{\text {neo/neo }}$. Mice with ubiquitous expression of CMV-Flp were mated with $D s g 2^{\text {neo/neo }}$ mice to remove the neomycin selection cassette, and mice with ubiquitous expression of CMV-Cre were used to remove the fourth and fifth exons of Dsg2 (Dsg $\left.2^{\mathrm{mut} / \mathrm{mut}}\right)$. Loss of exons 4 and 5 causes a frameshift mutation and premature termination of translation.

$J U P^{2157 \mathrm{del} 2}$ mutant mice. We generated a transgenic construct expressing $J U P^{2157 \mathrm{del} 2} \mathrm{cDNA}$ under the control of the cardiac-specific mouse $\alpha$-myosin heavy chain $(\alpha \mathrm{MHC})$ promoter, and a $300-b p$ DNA fragment containing bovine growth hormone polyadenylation sequences (bGHpA) was ligated $3^{\prime}$ to the 2.2-kb PLK cDNA open reading frame. The fragment containing PLK/bGHpA was then ligated downstream from the 5.5-kb mouse cardiac-specific aMHC promoter (provided by Jeffrey Robbins, Cincinnati Children's Hospital Medical Center, Cincinnati, Ohio, USA) and subjected to site-directed mutagenesis to introduce the $2157 \mathrm{del} 2$ mutation. Successful site-directed mutagenesis was confirmed by direct sequencing. A $6.8-\mathrm{kb}$ ScaI-KpnI DNA fragment was isolated, purified, and injected into the pronuclei of fertilized FVB mouse eggs (Beth Israel Deaconess Medical Center, Core Transgenic Mouse Facility). Transgenic mice harboring the $\alpha \mathrm{MHC}$ promoter/PLK cDNA were identified by PCR with genomic DNA prepared from tail biopsies. Routine genotyping was performed by PCR with the use of an upper primer (GCAGGCAGTACACCCTGAAAAA) from the $\alpha \mathrm{MHC}$ promoter and a lower primer (TGGACAGCTGGTTCACGATCAT) from the JUP CDNA to amplify a 400-bp fragment spanning the junction between the $\alpha \mathrm{MHC}$ promoter and the $J U P$ cDNA. Four independent founder lines were identified and mated to FVB WT mice to generate pure FVB genetic background WT and transgenic offspring. Levels of expression of the transgene were subsequently identified by Western immunoblotting on lysates prepared from cardiac samples. The transgene product migrates at a lower molecule weight to the WT endogenous protein due to the $2157 \mathrm{del} 2$ mutation. A single founder line was propagated where expression of the exogenous truncated PLK protein was at roughly equivalent levels to expression of the WT endogenous mouse PLK.

\section{Drug treatment}

At 6 months $\left(J U P^{2157 \text { del2 }}\right)$ and 3 weeks $\left(D s g 2^{\text {mut } / \text { mut }}\right)$ of age, mice were given daily i.p. injections of SB2 (2.5 $\mathrm{mg} / \mathrm{kg} / \mathrm{d})$ for $6\left(J U P^{2157 \mathrm{del} 2}\right)$ or 13 (Dsg2 $\left.2^{\mathrm{mut} / \mathrm{mut}}\right)$ weeks. Drug control mice were given equivalent volume/ $\mathrm{kg} / \mathrm{d}$ of vehicle (DMSO).

\section{Forced swim exercise experiments and tissue collection}

WT, $D s g 2^{\mathrm{mut} /+}$, and $D s g 2^{\mathrm{mut} / \mathrm{mut}}$ mice began SB2 treatment at 3 weeks of age, and a subset of these mice began graded exercise training (swimming) at 5 weeks of age mice (10 minutes per increment, 5 days per week, for 2 weeks). More specifically, mice began training at 5 weeks of age on a Monday, at which point they swam for 10 minutes. Each following day 10-minute increments would be added, such that by the end of the first 
training week (the first Friday) mice swam 50 minutes. No mice swam on Saturdays or Sundays. The second training week mice swam 50 minutes on Monday, and each following day 10-minute increments would be added. By the end of the second training week (the second Friday), mice swam 90 minutes. From the start of the third week (Monday, 7 weeks of age), all mice swam 90 minutes per day, 5 days per week. This exercise regimen was maintained until mice were 16 weeks of age. SB2 treatment was also maintained for the duration of the study. At treatment endpoint, sedentary and exercised mice were euthanized, and hearts were excised and analyzed for changes in histology.

\section{PCR and real-time RT-PCR analysis}

DNA was isolated from mouse tail lysates, and $30 \mathrm{ng} / \mu \mathrm{l}$ of DNA was run on a thermocycler (PTC-200, MJ Research). DNA primers specific for $D_{s g} 2^{\text {mut }} /$ mut were as follows: forward, 5'-GGTAGGAACAAGAAGATCAGT-3'; reverse, 5'-TCTAATAGCCAAGACTGAG-3'. RNA was isolated from ventricular myocardia using Trizol (Invitrogen) and 1-Bromo-3-Chloropropane (Molecular Research Center Inc.), then reverse transcribed (High Capacity cDNA Reverse Transcription Kit, Applied Biosystems).

\section{Transthoracic echocardiography}

Transthoracic echocardiography was performed at endpoint in nonsedated mice using a 2100 Visualsonic, equipped with an ultrahigh frequency linear array microscan transducer of $40 \mathrm{MHz}$. Measurements were performed according to the American Society of Echocardiography guidelines. M-mode echocardiograms were acquired from the parasternal long-axis view of the LV at the level of the papillary muscles and at a sweep speed of $200 \mathrm{~mm} / \mathrm{sec}$. Using the M-mode echocardiogram image, the following parameters were derived: (a) LV posterior wall thickness at end of diastole (LVPWTd) and systole (LVPWTs), (b) interventricular septal thickness at end of diastole (IVSd) and systole (IVSs), (v) LV chamber diameter at end of diastole (LVEDd), and (d) LV chamber diameter at end of systole (LVEDs). The FS represents the percentage change in LV chamber dimension and systolic contraction used to estimate LV wall contractility or the systolic function according to the following equation: FS $(\%)=[($ LVEDd - LVEDs $) / L V E D d] \times 100$. The LV mass was derived and used in the assessment of LV hypertrophy and enlargement using the following equation: $\mathrm{LV}$ mass $(\mathrm{mg})=1.055 \times\left[(\text { IVSd + LVEDd + PWTED })^{3}-(\text { LVEDd })^{3}\right]$, where 1.055 is the specific gravity of the myocardium and PWTED is the posterior wall thickness at end diastole. The individual performing (Djahida Bedja) the experiment was blinded. For each mouse, 3 to 5 values for each measurement were obtained and averaged for evaluation.

\section{Telemetry electrocardiography}

Following final echocardiography, mice were anesthetized and maintained via nose cone inhalation ( $2 \%$ isoflurane, in $95 \% \mathrm{O}_{2}$ and $5 \% \mathrm{CO}_{2}$ ), and a subcutaneous electrocardiographic (ECG) transmitter was sutured in place (7-0 Ethicon) following the manufacture's protocol (DSI PhysioTel ETA-F10) to obtain Lead I ECG recordings. Mice were allowed to recover for 3 days, and mouse ECGs were recorded for 24 hours, 90 minutes, or 10 minutes in sedentary, exercised, and double-mutant mice, respectively. ECG parameters, such as S-amplitude, PR, RR, and QRS intervals, were analyzed following previously described guidelines for murine ECG morphologies (35). In sedentary studies, average QRS duration was obtained in addition to the change in ventricular ectopy (QRS $\times$ frequency). Individuals who analyzed the ECG telemetry data (Deeptankar DeMazumder, Andre G. Kleber) were blinded.

\section{Western blot analysis}

Ventricular myocardial tissue was lysed in RIPA buffer (containing phosphatase and proteinase inhibitor cocktail, Sigma-Aldrich), total protein content was quantified (Pierce BCA Assay Kit), and $20 \mu \mathrm{g}$ protein lysates were separated on a polyacrylamide gel. After wet transfer, blots were blocked with 5\% nonfat milk, immunostained with primary (overnight, $4^{\circ} \mathrm{C}$ ) and then secondary antibodies ( 1 hour) and analyzed using the LI-COR Odyssey imaging system.

\section{Analysis of immunostained myocardium}

Formalin-fixed, paraffin-embedded myocardium was cut at a 5- $\mu \mathrm{m}$ thickness and mounted on clear, plus microscope slides. Sections were deparaffinized with xylene, rehydrated via 3 ethanol washes, and processed for antigen retrieval. Following antigen retrieval, sections were permeabilized, bleached and blocked 
in $0.5 \%$ Triton-X, $3 \%$ hydrogen peroxide, and $5 \%$ bovine serum albumin, respectively, for 1 hour at room temperature. Sections were stained with primary antibodies overnight at $4^{\circ} \mathrm{C}$ : mouse monoclonal antiDSG2 (6D8, Santa Cruz sc-53486), goat polyclonal anti-DSG2 (V-19, Santa Cruz sc-14860), mouse monoclonal anti-N-cadherin (13A9, Santa Cruz sc-55987), rabbit polyclonal anti-N-cadherin (SigmaAldrich C3678), rabbit polyclonal anti-GAPDH (FL-335, Santa Cruz sc-25778), rabbit polyclonal antiPLK (Thermo Fisher PA5-29930), mouse monoclonal anti-PLK (Sigma-Aldrich P8087), mouse monoclonal anti-desmoplakin (Fitzgerald 10R-D109A), mouse monoclonal anti-SAP97 (Santa Cruz sc-9961), rabbit polyclonal anti-Cx43 (Sigma-Aldrich C6219), mouse monoclonal anti-Cx43 (Millipore MAB3068), rabbit polyclonal anti-GSK3 $\beta$ (27C10, Cell Signaling 9315S), rabbit monoclonal anti-GSK3 $\alpha$ (Cell Signaling 4337), and rabbit monoclonal anti-phosphoGSK3 $\beta$-S9 (Cell Signaling 5558). Additional sections were stained for TUNEL, according to the manufacturer's instructions (R\&D Systems Inc., Kit 4810). Slides were incubated with host-specific secondary antibodies and cover slipped with mounting media (Invitrogen, ProLong Gold with Dapi), and immunoreactive signal was detected via laser scanning microscopy (Zeiss LSM 510 Meta). Sections were stained with Masson's trichrome (MTC) and H\&E, and images were detected on an Olympus BX51TF and photographed with a DP70 color camera (Olympus). H\&E- and MTC-stained myocardium were scored, as previously described (36), via blinded analysis (Peter Andersen).

\section{Engineered microprinting technique in NRVM cultures}

Control and mutant ACM expressing NRVMs were harvested as paired myocytes and grown on lines of fibronectin (width $15 \mu \mathrm{m}$ ) stamped on a PDMS substrate using soft microlithography as previously described (37-39).

\section{CSK3 $\beta$ shRNA transfection in NRVM cultures expressing mutant ACM constructs} NRVM cultures were transfected with GSK3 $\beta$ shRNA virus (courtesy of Junichi Sadoshima, Department of Cell Biology and Molecular Medicine, New Jersey Medical School, Newark, New Jersey, USA) 24 hours after isolation. Primary NRVM cultures were harvested from 1-day-old Wistar rat pups (strain 003, Charles River Laboratories International Inc.). Following 96 hours of transfection, GSK3 $\beta$ shRNA NRVM cultures were then cotransfected with either $J U P^{2157 \text { del2 }}$ or $P K P 2^{1851 \text { del123 }}$ constructs for 24 hours. Cotransfected cultures were then analyzed for changes in protein expression and distribution and assessed via Western immunoblot and immunofluorescence, respectively. Cultures not transfected with any constructs as well as cultures transfected with GSK3 $\beta$ shRNA alone served as negative controls, while cultures transfected with either $J U P^{2157 \mathrm{del} 2}$ or $P K P 2^{1851 \mathrm{del123}}$ constructs served as positive controls.

\section{Statistics}

In all studies, at least 4 mice were analyzed for each condition. Data are presented as mean \pm SEM. $P<0.05$ was deemed statistically significant. As appropriate, associations between continuous dependent variables were analyzed using 2-tailed Student's paired/unpaired $t$ test (binary independent variables) or ANOVA (2 or more variables).

\section{Study approval}

The following experiments conformed to the Guide for the Care and Use of Laboratory Animals from the National Institutes of Health (NIH publication no. 85-23, revised 1996), and all protocols were approved by Harvard Medical School and Johns Hopkins School of Medicine. Mice were housed in a climate-controlled facility with a 12-hour-light/dark cycle and provided access to standard mouse chow and water ad libitum. GSK33-S9A mutant mice were provided courtesy of Eleonore Beurel (Department of Psychiatry and Behavioral Sciences, Miami School of Medicine, Miami, Florida, USA).

\section{Author contributions}

JES, DPJ, SPC, and AA designed and conceived the study; SPC, AA, NAA, PA, DB, DD, and RJ acquired data; SPC, AA, PA, DB, DD, and AGK analyzed data; CAM and RL provided critical expertise and interpretation of data; and SPC, AA, DPJ, and JES prepared and drafted the manuscript.

\section{Acknowledgments}

We would like to thank Eleonore Beurel (University of Miami, Miller School of Medicine) from whom we 
acquired the mutant GSK3ß-S9A mice and Junichi Sadoshima (Department of Cell Biology and Molecular Medicine, New Jersey Medical School) from whom we acquired the GSK3 $\beta$ shRNA construct. This work was supported by NIH grants R01 HL102361 (to J.E. Saffitz) and RCHL100110 (to J.E. Saffitz and C.A. MacRae) and Johns Hopkins University School of Medicine T32 cardiology training grant (David Kass); by an American Heart Association Grant-in-Aid (to J.E. Saffitz) and scientist development grant (to A. Asimaki); and by The Hartwell Foundation (to D.P. Judge), the Thomas Rutherfoord Foundation (to D.P. Judge), and the Magic That Matters Fund (to D.P. Judge).

Address correspondence to: Daniel P. Judge, Johns Hopkins School of Medicine, Department of Medicine/Cardiology, 720 Rutland Avenue, Baltimore, Maryland 21205, USA. Phone: 410.614.3085; E-mail: djudge@jhmi.edu. Or to: Jeffrey E. Saffitz, Harvard Medical School, Beth Israel Deaconess Med. Ctr., Department of Pathology, 330 Brookline Avenue, Boston, Massachusetts 02215, USA. Phone: 617.667.4343; E-mail: jsaffitz@bidmc.harvard.edu.

1. Sen-Chowdhry S, McKenna WJ. Sudden death from genetic and acquired cardiomyopathies. Circulation. 2012;125(12):1563-1576.

2. Garcia-Gras E, et al. Suppression of canonical wnt/beta-catenin signaling by nuclear plakoglobin recapitulates phenotype of arrhythmogenic right ventricular cardiomyopathy. J Clin Invest. 2006;116(7):2012-2021.

3. Asimaki A, et al. Identification of a new modulator of the intercalated disc in a zebrafish model of arrhythmogenic cardiomyopathy. Sci Transl Med. 2014;6(240):240ra74.

4. Eldar-Finkelman H, Martinez A. GSK-3 inhibitors: Preclinical and clinical focus on CNS. Front Mol Neurosci. 2011;4:32.

5. Coghlan MP, et al. Selective small molecule inhibitors of glycogen synthase kinase-3 modulate glycogen metabolism and gene transcription. Chem Biol. 2000;7(10):793-803.

6. Lal H, Ahmad F, Woodgett J, Force T. The GSK-3 family as therapeutic target for myocardial diseases. Circ Res. 2015;116(1):138-149.

7. Hirotani S, et al. Inhibition of glycogen synthase kinase 3beta during heart failure is protective. Circ Res. 2007;101(11):1164-1174

8. Woulfe KC, et al. Glycogen synthase kinase-3beta regulates post-myocardial infarction remodeling and stress-induced cardiomyocyte proliferation in vivo. Circ Res. 2010;106(10):1635-1645.

9. Miura T, Tanno M. Mitochondria and GSK-3 $\beta$ in cardioprotection against ischemia/reperfusion injury. Cardiovasc Drugs Ther. 2010;24(3):255-263.

10. Matsuda T, et al. Distinct roles of GSK-3 $\alpha$ and GSK-3 $\beta$ phosphorylation in the heart under pressure overload. Proc Natl Acad Sci US A. 2008;105(52):20900-20905.

11. Awad MM, et al. DSG2 mutations contribute to arrhythmogenic right ventricular dysplasia/cardiomyopathy. Am J Hum Genet. 2006;79(1):136-142.

12. Kirchhof P, et al. Age- and training-dependent development of arrhythmogenic right ventricular cardiomyopathy in heterozygous plakoglobin-deficient mice. Circulation. 2006;114(17):1799-1806.

13. Asimaki A, et al. A new diagnostic test for arrhythmogenic right ventricular cardiomyopathy. $N$ Engl J Med. 2009;360(11):1075-1084.

14. Ai Z, Fischer A, Spray DC, Brown AM, Fishman GI. Wnt-1 regulation of connexin43 in cardiac myocytes. J Clin Invest. 2000;105(2):161-171.

15. Li VS, et al. Wnt signaling through inhibition of $\beta$-catenin degradation in an intact Axin1 complex. Cell. 2012;149(6):1245-1256.

16. Richardson P, et al. Report of the 1995 World Health Organization/International Society and Federation of Cardiology Task Force on the Definition and Classification of cardiomyopathies. Circulation. 1996;93(5):841-842.

17. Asimaki A, et al. Altered desmosomal proteins in granulomatous myocarditis and potential pathogenic links to arrhythmogenic right ventricular cardiomyopathy. Circ Arrhythm Electrophysiol. 2011;4(5):743-752.

18. James CA, et al. Exercise increases age-related penetrance and arrhythmic risk in arrhythmogenic right ventricular dysplasia/ Cardiomyopathy-Associated desmosomal mutation carriers. J Am Coll Cardiol. 2013;62(14):1290-1297.

19. Sawant AC, et al. Exercise has a disproportionate role in the pathogenesis of arrhythmogenic right ventricular dysplasia/cardiomyopathy in patients without desmosomal mutations. J Am Heart Assoc. 2014;3(6):e001471.

20. Lal H, et al. Cardiac fibroblast glycogen synthase kinase- $3 \beta$ regulates ventricular remodeling and dysfunction in ischemic heart. Circulation. 2014;130(5):419-430.

21. McManus EJ, et al. Role that phosphorylation of GSK3 plays in insulin and wnt signalling defined by knockin analysis. EMBO J. 2005;24(8):1571-1583.

22. Gillers BS, et al. Canonical wnt signaling regulates atrioventricular junction programming and electrophysiological properties. Circ Res. 2015;116(3):398-406.

23. Eshkind L, Tian Q, Schmidt A, Franke WW, Windoffer R, Leube RE. Loss of desmoglein 2 suggests essential functions for early embryonic development and proliferation of embryonal stem cells. Eur J Cell Biol. 2002;81(11):592-598.

24. Krusche CA, et al. Desmoglein 2 mutant mice develop cardiac fibrosis and dilation. Basic Res Cardiol. 2011;106(4):617-633.

25. Lombardi R, da Graca Cabreira-Hansen M, Bell A, Fromm RR, Willerson JT, Marian AJ. Nuclear plakoglobin is essential for differentiation of cardiac progenitor cells to adipocytes in arrhythmogenic right ventricular cardiomyopathy. Circ Res. 2011;109(12):1342-1353.

26. Zhang Z, et al. Normalization of naxos plakoglobin levels restores cardiac function in mice. J Clin Invest. 2015;125(4):1708-1712.

27. El-Haou S, et al. Kv4 potassium channels form a tripartite complex with the anchoring protein SAP97 and CaMKII in cardiac myocytes. Circ Res. 2009;104(6):758-769. 
28. Petitprez S, et al. SAP97 and dystrophin macromolecular complexes determine two pools of cardiac sodium channels Nav1.5 in cardiomyocytes. Circ Res. 2011;108(3):294-304.

29. Milstein ML, et al. Dynamic reciprocity of sodium and potassium channel expression in a macromolecular complex controls cardiac excitability and arrhythmia. Proc Natl Acad Sci U S A. 2012;109(31):E2134-E2143.

30. Gillet L, et al. Cardiac-specific ablation of synapse-associated protein SAP97 in mice decreases potassium currents but not sodium current. Heart Rhythm. 2015;12(1):181-192.

31. Hariharan V, et al. Arrhythmogenic right ventricular cardiomyopathy mutations alter shear response without changes in cell-cell adhesion. Cardiovasc Res. 2014;104(2):280-289.

32. Swope D, Cheng L, Gao E, Li J, Radice GL. Loss of cadherin-binding proteins beta-catenin and plakoglobin in the heart leads to gap junction remodeling and arrhythmogenesis. Mol Cell Biol. 2012;32(6):1056-1067.

33. Sato PY, et al. Interactions between ankyrin-G, plakophilin-2, and Connexin 43 at the cardiac intercalated disc. Circ Res. 2011;109(2):193-201.

34. Fabritz L, et al. Load-reducing therapy prevents development of arrhythmogenic right ventricular cardiomyopathy in plakoglobin-deficient mice. J Am Coll Cardiol. 2011;57(6):740-750

35. Boukens BJ, Rivaud MR, Rentschler S, Coronel R. Misinterpretation of the mouse ECG: 'musing the waves of mus musculus'. J Physiol (Lond). 2014;592(21):4613-4626.

36. Andrassy M, et al. High-mobility group box-1 in ischemia-reperfusion injury of the heart. Circulation. 2008;117(25):3216-3226.

37. Beauchamp P, et al. Electrical coupling and propagation in engineered ventricular myocardium with heterogeneous expression of connexin43. Circ Res. 2012;110(11):1445-1453.

38. Geisse NA, Sheehy SP, Parker KK. Control of myocyte remodeling in vitro with engineered substrates. In Vitro Cell Dev Biol Anim. 2009;45(7):343-350.

39. Singhvi R, et al. Engineering cell shape and function. Science. 1994;264(5159):696-698 